\title{
Development of a B-cell maturation antigen-specific T-cell antigen coupler receptor for multiple myeloma
}

\author{
Ksenia Bezverbnaya ${ }^{1}$, Duane Moogk ${ }^{1}$, Derek Cummings ${ }^{1}$, Christopher L. Baker ${ }^{1}$, Craig Aarts ${ }^{1}$, \\ Galina Denisova ${ }^{1}$, Michael Sun ${ }^{1}$, Jamie D. McNicol ${ }^{1}$, Rebecca C. Turner ${ }^{1}$, Anthony F. Rullo ${ }^{1}$, \\ S. Ronan Foley ${ }^{1,2}$, Jonathan L. Bramson ${ }^{1, *}$ \\ ${ }^{1}$ Department of Pathology and Molecular Medicine, McMaster Immunology Research Centre, McMaster University, Hamilton, Canada \\ 2 Juravinski Hospital and Cancer Centre, Hamilton, Canada
}

\section{A R T I C L E I N F O}

\section{Article History:}

Received 31 January 2021

Accepted 14 May 2021

\section{Key Words:}

BCMA

engineered $\mathrm{T}$ cells

immunotherapy

multiple myeloma

\begin{abstract}
A B S T R A C T
Background aims: T cells engineered with synthetic receptors have delivered powerful therapeutic results for patients with relapsed/refractory hematologic malignancies. The authors have recently described the T-cell antigen coupler (TAC) receptor, which co-opts the endogenous T-cell receptor (TCR) and activates engineered $\mathrm{T}$ cells in an HLA-independent manner. Here the authors describe the evolution of a next-generation TAC receptor with a focus on developing a TAC-engineered T cell for multiple myeloma.

Methods: To optimize the TAC scaffold, the authors employed a bona fide antigen-binding domain derived from the B-cell maturation antigen-specific monoclonal antibody C11D5.3, which has been used successfully in the clinic. The authors first tested humanized versions of the UCHT1 domain, which is used by the TAC to co-opt the TCR. The authors further discovered that the signal peptide affected surface expression of the TAC receptor. Higher density of the TAC receptor enhanced target binding in vitro, which translated into higher levels of Lck at the immunological synapse and stronger proliferation when only receptor-ligand interactions were present. Results: The authors observed that the humanized UCHT1 improved surface expression and in vivo efficacy. Using TAC T cells derived from both healthy donors and multiple myeloma patients, the authors determined that despite the influence of receptor density on early activation events and effector function, receptor density did not impact late effector functions in vitro, nor did the receptor density affect in vivo efficacy.

Conclusions: The modifications to the TAC scaffold described herein represent an important step in the evolution of this technology, which tolerates a range of expression levels without impacting therapeutic efficacy. (C) 2021 International Society for Cell \& Gene Therapy. Published by Elsevier Inc. This is an open access article under the CC BY-NC-ND license (http://creativecommons.org/licenses/by-nc-nd/4.0/)
\end{abstract}

\section{Introduction}

Multiple myeloma (MM) is an incurable plasma cell cancer with a poor prognosis for relapsed/refractory disease and limited treatment options. The introduction of immunomodulatory drugs into the MM treatment landscape brought major improvements to overall patient survival [1-3] and supported the use of immune-based approaches for MM. Recently, the growing number of immunotherapeutic agents entering clinical testing has expanded to cell-based therapies, monoclonal antibodies, antibody-drug conjugates and bispecific T-cell engagers [4-6].

\footnotetext{
* Correspondence: Jonathan L. Bramson, PhD, Department of Pathology and Molecular Medicine, McMaster Immunology Research Centre, McMaster University, 1280 Main St West, HSC 2E17, Hamilton L8S 4K1, Canada.

E-mail address: bramsonj@mcmaster.ca (J.L. Bramson).
}

Common targets for MM immunotherapeutics include B-cell maturation antigen (BCMA), CD38, CD138, SLAMF7, checkpoint molecules (e. g., programmed cell death protein 1 [PD-1] and cytotoxic T lymphocyte-associated protein 4) and their ligands, vascular endothelial growth factor and IL-6 [7]. Among these targets, BCMA stands out because of its biological role in MM cell survival and proliferation [8-10], high expression levels on malignant plasma cells and lack of expression in non-hematopoietic tissues [11]. Several BCMA-specific products have already shown clinical efficacy in patients with relapsed/ refractory MM. The antibody-drug conjugate GSK2857916 demonstrated a $60 \%$ overall response rate [12], whereas the bispecific T-cell engager AMG 420 showed a 70\% response rate at the highest tolerable dose [13]. Responses in currently completed or ongoing trials with Tcell-based therapeutics range from $64 \%$ to $100 \%$ [14-23], highlighting the susceptibility of previously treated MM to BCMA-targeting agents. 
T-cell immunotherapies for MM include antigen-specific endogenous [24], T-cell receptor (TCR)-engineered [25,26] and chimeric antigen receptor (CAR)-engineered T cells [15-23,27,28]. Because of their customizable structure, lack of dependence on HLA matching, scalable manufacturing and clinical efficacy in other hematologic malignancies, CAR-engineered T (CAR T) cells have been leading the development in T-cell therapies for MM. Specifically, autologous BCMA-specific secondgeneration CAR T cells have shown encouraging levels of efficacy. Interestingly, baseline BCMA expression does not correlate with response $[16,29]$. Data from clinical trials with the longest follow-ups indicate that despite the high likelihood of initial response, most patients will eventually progress or relapse $[15,16]$. However, unlike tumor relapses following CD19-CAR T-cell therapy, which are characterized by antigen loss or alternative splicing [30-32], most relapsed MM tumors remain BCMA-positive [17,33]. This suggests that the potency of the engineered T-cell product can be improved, and alternative modes of engineering are one way to address this issue.

The authors' lab has taken a TCR-centric approach to T-cell engineering and created a synthetic receptor that the authors have termed the T-cell antigen coupler (TAC) [34], which redirects endogenous TCRs to the target of interest and effectively combines HLA-independent antigen recognition with the canonical mechanisms of T-cell activation and regulation. In the authors' previous experience, $\mathrm{T}$ cells engineered with TAC receptors (TAC $T$ cells) have shown improved tolerability and enhanced discrimination between on-target and off-target responses compared with second-generation CAR T cells. Since TAC T cells may offer advantages over CAR T cells in the setting of human myeloma, the authors were keen to design a TAC receptor for human testing. In this article, the authors describe the optimization of a TAC scaffold carrying a BCMA-binding domain that has been proven clinically: the C11D5.3 single-chain variable fragment (scFv) [16]. The authors' experience shows that BCMA-TAC T cells derived from healthy and patient donors are efficacious against disseminated multiple myeloma xenografts. Moreover, the authors demonstrate that different surface expression levels of BCMA-TAC receptors do not influence in vivo TAC T-cell efficacy despite differences in in vitro performance.

\section{Methods}

\section{Cell lines}

Human myeloma cell lines KMS-11 and MM.1S (kindly provided by Dr Kelvin Lee, Roswell Park Cancer Institute, Buffalo, NY, USA) were cultured in RPMI 1640 (Gibco, Waltham, MA, USA) supplemented with $10 \%$ fetal bovine serum (FBS) (Gibco), 2 mM L-glutamine (BioShop Canada Inc, Burlington, ON, Canada), 10 mM 4-(2-hydroxyethyl)-1-piperazineethanesulfonic acid (HEPES) (Roche Diagnostics, Basel, Switzerland), $1 \mathrm{mM}$ sodium pyruvate (Sigma-Aldrich, St Louis, MO, USA), $1 \mathrm{mM}$ nonessential amino acids (Gibco), $100 \mathrm{U} / \mathrm{mL}$ penicillin (Gibco) and $100 \mu \mathrm{g} /$ $\mathrm{mL}$ streptomycin (Gibco). To generate luciferase-expressing cell lines, parental KMS-11 and MM.1S cell lines were transduced with lentivirus encoding enhanced firefly luciferase [35] as well as puromycin $\mathrm{N}$-acetyltransferase at a multiplicity of infection of 10 and selected in culture media supplemented with $8 \mu \mathrm{g} / \mathrm{mL}$ puromycin (InvivoGen, San Diego, CA, USA). HEK293T cells were cultured in Dulbecco's Modified Eagle's Medium (Gibco) supplemented with 10\% FBS (Gibco), 2 mM L-glutamine (BioShop Canada Inc), $10 \mathrm{mM}$ HEPES (Roche Diagnostics), $100 \mathrm{U} / \mathrm{mL}$ penicillin (Gibco) and $100 \mu \mathrm{g} / \mathrm{mL}$ streptomycin (Gibco) or $0.1 \mathrm{mg} / \mathrm{mL}$ Normocin (InvivoGen). All cells were cultured at $37^{\circ} \mathrm{C}, 95 \%$ ambient air and 5\% carbon dioxide.

\section{Receptor generation and lentivirus production}

TAC receptor sequence was constructed as described by Helsen et al. [34], and UCHT1 TAC variants were generated as described by patent WO2019071358 [36]. Human CD8 $\alpha$ signal peptide and murine
IgK signal peptide were used with the TAC constructs. C11D5.3 scFv complementary DNA (sequence from patent US20150051266 A1) was synthesized at GenScript. Lentivirus production was performed as described by Hammill et al. [37]. Briefly, $12 \times 10^{6}$ HEK293T cells plated on a 15-cm dish (Nunc, Rochester, NY, USA) were transfected with plasmids pRSV-Rev $(6.25 \mu \mathrm{g})$, pMD2.G (9 $\mu \mathrm{g})$, pMDLg-pRRE $(12.5 \mu \mathrm{g})$ and pCCL $(32 \mu \mathrm{g})$ using Opti-MEM (Gibco) and Lipofectamine 2000 (Thermo Fisher Scientific). At 12-16 h post-transfection, media were exchanged for media supplemented with $1 \mathrm{mM}$ sodium butyrate (Sigma-Aldrich). Supernatants were harvested 24-36 h later, and viral particles were concentrated by centrifugation using an Amicon filter system (MilliporeSigma, Burlington, MA, USA) and stored at $-80^{\circ} \mathrm{C}$. Viral titer $(\mathrm{TU} / \mathrm{mL})$ was determined post-thaw by serial dilution, transduction of HEK293T cells and enumeration of percent nerve growth factor receptor-positive $\left(\mathrm{NGFR}^{+}\right)$cells by flow cytometry.

\section{Engineering of human T cells}

The work described herein has been carried out in accordance with The Code of Ethics of the World Medical Association (Declaration of Helsinki) for experiments involving humans. Peripheral blood mononuclear cells (PBMCs) were obtained from healthy donors and MM patients who provided informed written consent in accordance with the Hamilton Integrated Research Ethics Board (ON, Canada). In some cases, PBMCs were collected from commercial leukapheresis products (HemaCare, Northridge, CA, USA, and StemCell Technologies, Vancouver, BC, Canada). PBMCs were isolated by Ficoll-Paque Plus gradient centrifugation (GE Healthcare, Chicago, IL, USA) and cryopreserved in inactivated human AB serum (Corning, Corning, NY, USA) containing 10\% dimethyl sulfoxide (Sigma-Aldrich) (healthy donors) or RPMI (Gibco) containing 12.5\% human serum albumin (Sigma-Aldrich) and 10\% dimethyl sulfoxide (MM donors).

PBMCs were stimulated with anti-CD3/28 Dynabeads (Gibco) at a 0.8:1 bead:cell ratio and cultured in RPMI 1640 (Gibco) containing 10\% FBS (Gibco), 2 mM L-glutamine (BioShop Canada Inc), $10 \mathrm{mM}$ HEPES (Roche Diagnostics), $1 \mathrm{mM}$ sodium pyruvate (Sigma-Aldrich), $1 \mathrm{mM}$ non-essential amino acids (Gibco), $55 \mu \mathrm{M} \beta$-mercaptoethanol (Gibco), $100 \mathrm{U} / \mathrm{mL}$ penicillin (Gibco), $100 \mu \mathrm{g} / \mathrm{mL}$ streptomycin (Gibco), $100 \mathrm{IU} / \mathrm{mL}$ rhL-2 and $10 \mathrm{ng} / \mathrm{mL}$ rhIL-7 (PeproTech). Cells were transduced with lentivirus $16-24 \mathrm{~h}$ later at a multiplicity of infection of 2. Dynabeads were removed on day 4 of culture. Cells were sorted using an EasySep human CD271 positive selection kit (StemCell Technologies) on day 6 to day 7 of culture and expanded for a total culture period of 14 days prior to use in vitro and/or in vivo. For in vivo experiments, T cells were cryopreserved in CryoStor CS10 (StemCell Technologies) according to the manufacturer's instructions. The details of the vector copy number assessment are provided in the supplementary material.

\section{In silico modeling}

Protein docking was modeled using web-based ClusPro 2.0 software (Boston University, Boston, MA, USA) [38], and the models were analyzed using web-based contacts of structural units software (Weizmann Institute of Science, Rehovot, Israel) [39] for the number of contacts between proteins of interest. All predicted bonds for each amino acid were added and displayed as the total number of contacts.

\section{Protein production and purification}

UCHT1 scFv variants were cloned into pET-20b(+) to add a C-terminal His-tag and transformed into competent Escherichia coli BL21 (DE3) (New England Biolabs, Ipswich, MA, USA). All recombinant strains were grown at $37^{\circ} \mathrm{C}$ to $\mathrm{OD}_{600}$ of approximately 0.6 in 
Luria-Bertani medium with $50 \mu \mathrm{g} / \mathrm{mL}$ ampicillin (Sigma-Aldrich), and protein expression was induced using $0.04 \mathrm{mM}$ isopropyl $\beta$-D-1thiogalactopyranoside (Sigma-Aldrich). After a 4-h induction at $37^{\circ} \mathrm{C}$, cells were harvested by centrifugation at $5000 \times \mathrm{g}$ for $10 \mathrm{~min}$, and pellets were lysed using BugBuster master mix (MilliporeSigma) in the presence of cOmplete protease inhibitor cocktail (Roche). Denaturing buffer (phosphate-buffered saline [PBS], $8 \mathrm{M}$ urea, $500 \mathrm{mM}$ sodium chloride $[\mathrm{NaCl}]$ ) was added to the cellular debris to extract recombinant protein from the inclusion bodies. After 40-min denaturation, samples were centrifuged and loaded onto 1-mL HiTrap chelating columns (Cytiva, Marlborough, MA, USA) charged with $100 \mathrm{mM}$ nickel. Denatured protein was refolded using a decreasing gradient of urea (PBS, 6-0 M urea, $500 \mathrm{mM} \mathrm{NaCl}$ ). The proteins were eluted off the column with an increasing gradient of imidazole (PBS, 0-250 $\mathrm{mM}$ imidazole, $500 \mathrm{mM} \mathrm{NaCl}$ ). Sodium dodecyl sulfate-polyacrylamide gel electrophoresis was used to determine which fraction contained the protein. The fraction was then run through a PD-10 desalting column (Cytiva) and eluted in PBS to remove salt and imidazole ions. Purified recombinant proteins were concentrated using 10-KDa Amicon Ultra 0.5 centrifugal filter units (MilliporeSigma) and quantified using a Bradford assay (Bio-Rad Laboratories, Hercules, CA, USA).

\section{Determination of the binding constant using bio-layer interferometry}

Bio-layer interferometry assays were performed on the Octet Red96 system (ForteBio, Menlo Park, CA, USA). The scFv proteins were dissolved in kinetics buffer (PBS with a pH of 7.4, Tween 20 at $0.02 \%, 0.1 \%$ bovine serum albumin) to create a dilution series $(1.25-500 \mathrm{nM})$. Probes were pre-wet for 600 seconds, and $100 \mathrm{nM}$ of biotinylated human $\mathrm{CD} 3 \varepsilon \delta$ heterodimer (ACROBiosystems, Newark, DE, USA) was loaded for 240 seconds onto streptavidin biosensors (ForteBio) and quenched in 5\% milk. Association with the scFvs lasted 180 seconds, followed by 600-second dissociation in kinetics buffer. The data were analyzed in Prism 8 (GraphPad Software, San Diego, CA, USA) with pre-installed binding kinetics formulas for one phase exponential decay and one concentration of hot ligand to estimate the binding constant for the scFvs.

\section{Flow cytometry}

Surface expression of TAC constructs was determined by staining with recombinant human BCMA-Fc protein (category no. 193-BC; R\&D Systems, Minneapolis, MN, USA) followed by phycoerythrin (PE)-conjugated goat anti-human IgG (category no. 109-115-098; Jackson ImmunoResearch, West Grove, PA, USA). Other phenotypic markers were detected with Pacific Blue-conjugated mouse antihuman CD4 (category no. 558116; BD Pharmingen, San Diego, CA, USA), Alexa Fluor 700-conjugated mouse anti-human $\mathrm{CD} 8 \alpha$ (category no. 56-0086-82; eBioscience, San Diego, CA, USA) and VioBright fluorescein isothiocyanate (FITC)-conjugated mouse anti-human NGFR (category no. 130-104-893; Miltenyi Biotec).

Binding of purified His-tagged scFvs was detected by staining PBMCs with scFvs followed by mouse anti-6X His (category no. 552565; BD Pharmingen), PE-conjugated goat anti-mouse IgG (category no. 115-116-146; Jackson ImmunoResearch) and PerCP-Cy5.5conjugated mouse anti-human CD8 $\alpha$ (category no. 45-0088-41; eBioscience) and Alexa Fluor 700-conjugated mouse anti-human CD4 (category no. 56-0048-41; eBioscience). Flow cytometry data were collected with a BD LSRFortessa or BD LSR II cytometer (BD Biosciences) and analyzed using FlowJo X software (BD Biosciences).

\section{Analysis of the time course of checkpoint receptor expression}

A total of $0.5 \times 10^{6}$ engineered T cells were stimulated with KMS11 tumor targets at a $1: 1$ effector:target $(\mathrm{E}: \mathrm{T})$ ratio at $37^{\circ} \mathrm{C}$ for the indicated time periods or left non-stimulated. Cells were stained with Live/Dead fixable near-infrared (near-IR) stain (Invitrogen) followed by BCMA-Fc protein (R\&D Systems) and PE-conjugated goat anti-human IgG (Jackson ImmunoResearch), PerCP-Cy5.5-conjugated mouse anti-human CD8 $\alpha$ (category no. 45-0088-41; eBioscience), Alexa Fluor 700-conjugated mouse anti-human CD4 (category no. 56-0048-41; eBioscience), VioBright FITC-conjugated mouse antihuman NGFR (Miltenyi Biotec), BV510-conjugated mouse antihuman CD138 (category no. 356518; BioLegend, San Diego, CA, USA), BV650-conjugated mouse anti-human CD69 (category no. 563835; BD Pharmingen), BV421-conjugated mouse anti-human PD-1 (category no. 562516; BD Pharmingen), Alexa Fluor 647-conjugated mouse anti-human lymphocyte activation gene 3 (LAG-3) (category no. 565716; BD Pharmingen) and BV785-conjugated mouse antihuman T-cell immunoglobulin and mucin domain 3 (TIM-3) (category no. 345032; BioLegend). Flow cytometry data acquisition was performed as indicated earlier and pre-processed using FlowJo X software.

The flow cytometry data were quality-checked using the flowAI Bioconductor package for $\mathrm{R}$ [40]. Detection and removal of anomalies provided the events for further pre-processing. Pre-gated viable, single-cell, CD138-NGFR+ events were randomly subsampled to 20000 cells and subjected to a differential analysis pipeline scripted in $\mathrm{R}$ (cran.r-project.org). Unsupervised clustering analysis was performed using flowSOM [41], and differential analysis was performed using diffcyt [42]. Tests for differential abundance of cell populations, represented by flowSOM meta-clusters, were calculated using the edgeR method. Tests for differential states in the expression of TAC, CD69, PD-1, LAG-3 and TIM-3 within these cell populations were calculated using the limma method. Data were visualized using t-distributed stochastic neighbor embedding [43]. The CATALYST package [44] was instrumental in providing a framework for passing data objects from one Bioconductor package to another and doing quality checks along the pipeline.

\section{Cytokine analysis}

A total of $4 \times 10^{5}$ engineered T cells were stimulated with $2 \times 10^{5}$ KMS-11, MM.1S or HEK293T cells for $4 \mathrm{~h}$ at $37^{\circ} \mathrm{C}$ in the presence of brefeldin A (BD GolgiPlug, category no. 555029; BD Pharmingen) and monensin (BD GolgiStop, category no. 554724; BD Pharmingen). Patient-derived engineered $\mathrm{T}$ cells were stimulated at a ratio of $10^{5}$ engineered T cells to $10^{5}$ target cells. Cells were stained for surface expression of CD4 (BD Pharmingen) and CD8 (eBioscience), fixed and permeabilized in Cytofix/Cytoperm buffer (category no. 554714; BD Pharmingen) and stained with allophycocyanin (APC)-conjugated mouse anti-human interferon gamma (IFN- $\gamma$ ) (category no. 554702; BD Pharmingen), PE-conjugated rat anti-human IL-2 (category no. 554566; BD Pharmingen) and FITC-conjugated mouse anti-human tumor necrosis factor alpha (TNF- $\alpha$ ) (category no. 554512; BD Pharmingen). Flow cytometry data were acquired and analyzed as indicated earlier.

For multiplex secreted cytokine analysis, $5 \times 10^{5}$ engineered T cells were stimulated with $5 \times 10^{5} \mathrm{KMS}-11$ cells for $24 \mathrm{~h}$ at $37^{\circ} \mathrm{C}$. Supernatants were spun down to remove cellular debris and analyzed in duplicate by Eve Technologies (Calgary, AB, Canada) with a 14-plex human high sensitivity T-cell discovery array (category no. HDHSTC14). Data were processed with Prism 8, and paired $t$-tests were performed to determine significance.

\section{In vitro cytotoxicity assay}

Engineered T cells were stimulated with $5 \times 10^{4}$ luciferaseexpressing cells in triplicate at the indicated E:T ratios for $8 \mathrm{~h}$ at $37^{\circ} \mathrm{C}$. Following stimulation, $0.15 \mathrm{mg} / \mathrm{mL}$ D-luciferin (category no. 122799; PerkinElmer, Waltham, MA, USA) was added and luminescence was 
measured with an open filter using a SpectraMax i3 plate reader (Molecular Devices, San Jose, CA, USA). Percent cytotoxicity was calculated using the following formula:

$\%$ Cytotoxicity $=\left(1-\frac{\text { Test Sample }- \text { Media Alone }}{\text { Tumor Alone }- \text { Media Alone }}\right) \times 100 \%$

\section{Proliferation assay}

Engineered T cells were labeled with CellTrace Violet dye (Invitrogen) and stimulated with KMS-11 or MM.1S tumor targets at an E:T ratio of 2:1 or left non-stimulated. For bead stimulations, protein G-coated polystyrene beads (Spherotech Inc, Lake Forest, IL, USA) were pre-coated with different concentrations of recombinant human BCMA-Fc protein (R\&D Systems) overnight at $4^{\circ} \mathrm{C}$ prior to the start of the assay. T cells were stimulated with beads at a 1:1 E:T ratio. All proliferation assay samples were incubated for 4 days at $37^{\circ} \mathrm{C}$ and stained with Live/Dead fixable near-IR stain (Invitrogen), PerCP-Cy5.5-conjugated mouse anti-human CD8 $\alpha$ (eBioscience), Alexa Fluor 700-conjugated mouse anti-human CD4 (eBioscience), VioBright FITC-conjugated mouse antihuman NGFR (Miltenyi Biotec) and APC-conjugated mouse antihuman CD138 (category no. 356505; BioLegend). Flow cytometry data were acquired as indicated earlier. Results were analyzed with FCS Express 6 (De Novo Software, Pasadena, CA, USA) by determining the starting generation peak based on the non-stimulated sample and using the software proliferation package for fitting a proliferation model and collecting corresponding statistics, such as percent divided.

\section{Conjugation assay}

Engineered T cells were pre-labeled with BV421-conjugated mouse anti-human NGFR (category no. 562562; BD Pharmingen), and myeloma cells were pre-labeled with APC-conjugated mouse anti-human CD138 (BioLegend). A total of $2.5 \times 10^{5} \mathrm{~T}$ cells and $2.5 \times 10^{5}$ tumor cells were briefly spun down; incubated for $0 \mathrm{~min}$, $10 \mathrm{~min}, 20 \mathrm{~min}$ and $40 \mathrm{~min}$ at $37^{\circ} \mathrm{C}$; fixed with $2 \%$ formaldehyde; and analyzed by flow cytometry without filtering or resuspension to preserve cell-cell conjugates.

\section{Nur77 and caspase-3 activation assessments}

A total of $5 \times 10^{5}$ engineered T cells and $5 \times 10^{5}$ tumor cells per sample were incubated for $2 \mathrm{~h}$ and $4 \mathrm{~h}$ at $37^{\circ} \mathrm{C}$. Cells were stained for surface expression of CD4 (BD Pharmingen), CD8 (eBioscience), CD138 (BioLegend), NGFR (BD Pharmingen) and CD69 (BD Pharmingen); fixed and permeabilized in Cytofix/Cytoperm buffer (BD Pharmingen); and stained with PE-conjugated mouse anti-mouse Nur77 (category no. 12-5965-82; eBioscience).

T cells were stimulated as in the Nur77 assay for 4 h, 8 h, 28 h, 52 $\mathrm{h}$ and $124 \mathrm{~h}$, stained with Live/Dead fixable near-IR stain (Invitrogen), then for surface markers as in the Nur77 assay, fixed, permeabilized, and stained with PE-conjugated active caspase-3 apoptosis kit (category no. 550914; BD Pharmingen) according to manufacturer's instructions. Flow cytometry data were acquired and analyzed as indicated earlier.

\section{Assembly of supported lipid bilayers and T-cell binding}

Lipid preparation and assembly of supported lipid bilayers were performed as described by Bertolet et al. [45] using lipids composed of 1,2-dioleoyl-sn-glycero-3-phosphocholine and 1,2-dioleoyl-snglycero-3-[(N-(5-amino-1-carboxypentyl) iminodiacetic acid) succinyl] (Avanti Polar Lipids Inc, Alabaster, AL, USA). Supported lipid bilayers were assembled in six-lane $\mu$-Slide VI 0.4 chambers (ibidi) and functionalized with recombinant human BCMA and human intercellular adhesion molecule 1 (ICAM-1) proteins, each containing C-terminal hIgG-His-tags (Novus Biologicals, Littleton, CO, USA), at densities of 250 molecules $/ \mu \mathrm{m}^{2}$ and 100 molecules $/ \mu \mathrm{m}^{2}$, respectively. The necessary protein concentration was determined by coating silica beads (Bangs Laboratories Inc, Fishers, IN, USA) with the lipids followed by titrated His-tagged protein concentrations and labeling with Alexa Fluor 647-labeled BCMA- or ICAM-1-specific antibodies (BioLegend). Silica beads were analyzed by flow cytometry concurrently with Quantum Alexa Fluor 647 MESF beads (Bangs Laboratories), and protein density on beads was determined using calibration curves created from the MESF beads and the effective F:P ratio of the Alexa Fluor 647-labeled antibodies using Simply Cellular anti-mouse IgG beads (Bangs Laboratories).

A total of $2 \times 10^{5} \mathrm{~T}$ cells in $100 \mu \mathrm{L}$ of media were pipetted in each flow chamber and incubated at $37^{\circ} \mathrm{C}$ for the indicated times. Cells were then immediately fixed with $100 \mu \mathrm{L}$ of Image-iT fixative solution (Thermo Fisher Scientific) for $20 \mathrm{~min}$ at room temperature, and unattached cells were removed by continuously flowing $3 \mathrm{~mL}$ of PBS through the flow chamber. Chambers were imaged on an Olympus CK40 inverted microscope, and the number of attached cells was determined by manual count of an entire field of view using an A10 PL $10 \times / 0.25$ objective.

\section{TAC T-cell immune synapse formation on supported lipid bilayers}

$\mathrm{T}$ cells were incubated on assembled lipid bilayers for the indicated times, fixed and washed as described earlier and permeabilized for $30 \mathrm{~min}$ at room temperature with PBS containing 5\% rat serum and $0.2 \%$ Triton X. Cells were washed via continuous flow of $3 \mathrm{~mL}$ of PBS and stained for $30 \mathrm{~min}$ at room temperature in BlockAid solution (Thermo Fisher Scientific) with Alexa Fluor Plus 405 phalloidin (category no. A30104; Thermo Fisher Scientific), Alexa Fluor 488-conjugated mouse anti-human CD11a/CD18 (category no. 363404; BioLegend) and Alexa Fluor 647-conjugated mouse anti-Lck (category no. 628304; BioLegend) or Alexa Fluor 647-conjugated mouse antihuman perforin (category no. 308110; BioLegend). Cells were finally washed via continuous flow of $2 \mathrm{~mL}$ PBS followed by the addition of $200 \mu \mathrm{L}$ of PBS containing $0.5 \% \mathrm{~N}$-propyl gallate. Images were acquired with a Leica DMI6000 B inverted microscope equipped with adaptive focus control, a motorized X-Y stage (MCL Micro-Drive; Mad City Labs Inc, Madison, WI, USA), a Leica $100 \times / 1.47 \mathrm{NA}$ oilimmersed TIRF objective and an Andor iXon Ultra EMCCD camera. Excitation was provided by 405-, 488- and 647-nm diode-pumped solid-state lasers (Spectral). Image analysis was performed using Fiji 2.0.0-rc-69/1.53p (ImageJ, Bethesda, MD, USA) [46]. All images were background subtracted. For Lck analysis, the area of actin exclusion central to the immune synapse was outlined manually and the integrated intensity of Lck was measured within that area. For perforin analysis, images were thresholded manually on perforin signal. Regions were created for any pixels above the threshold and transferred back to an unthresholded image, and the integrated perforin intensity within the regions was measured.

\section{In vivo studies}

All animal studies were conducted in accordance with McMaster Animal Research Ethics Board standards. Six- to 12-week-old NOD. Cg-Rag1tm1MomIl2rgtm1Wjl/SzJ mice bred in-house were injected intravenously with $10^{6} \mathrm{KMS}-11$ or MM.1S luciferase-expressing cells in $200 \mu \mathrm{L}$ PBS. Mice bearing 12-day-old KMS-11 or 15-day-old MM.1S tumors were treated intravenously with a single $200-\mu \mathrm{L}$ indicated dose of cryopreserved $\mathrm{TAC}^{+} \mathrm{T}$ cells. Tumor burden was monitored weekly by bioluminescence. Mice were injected intraperitoneally with $150 \mathrm{mg} / \mathrm{kg}$ D-luciferin (PerkinElmer), rested at room temperature for $14 \mathrm{~min}$ and imaged using an IVIS spectrum imager (Caliper Life Sciences, Waltham, MA, USA). Images were 
A

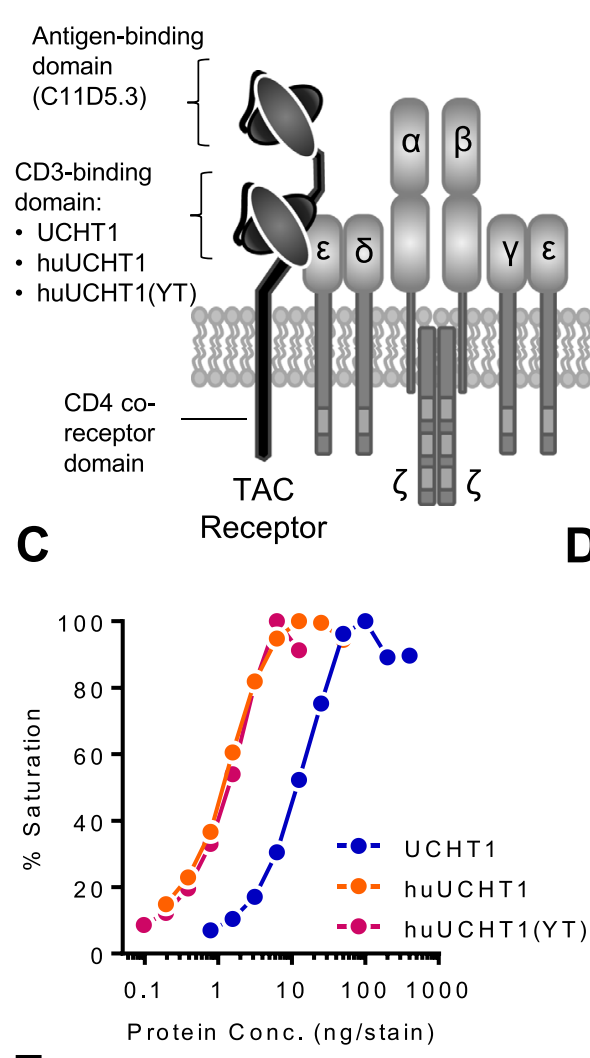

$\mathbf{E}$

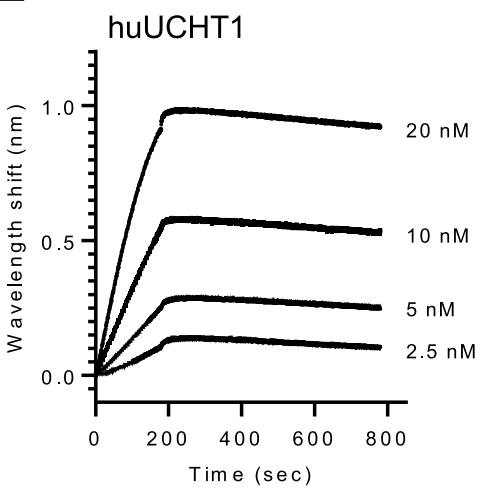

B

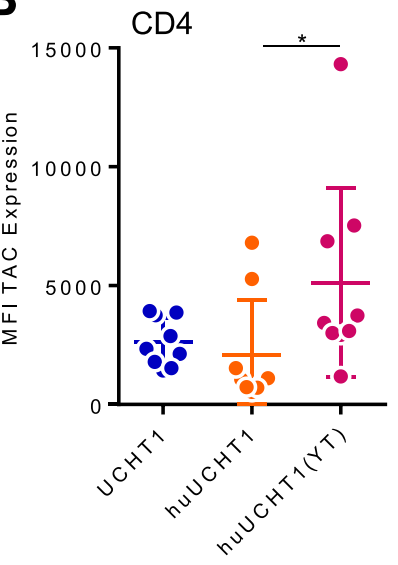

D

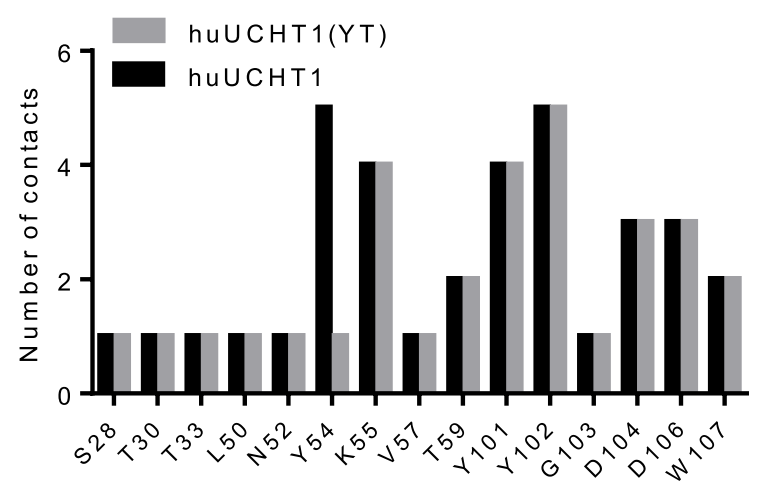

Am ino acid residues (heavy chain)

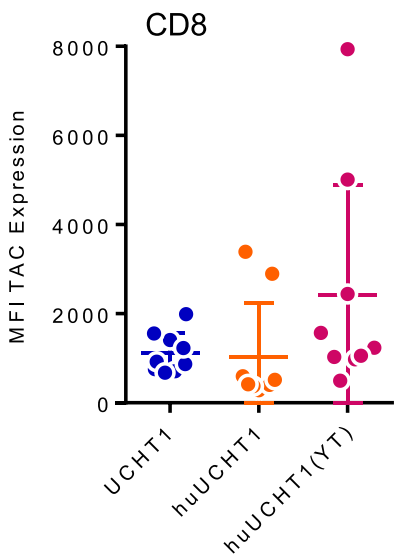

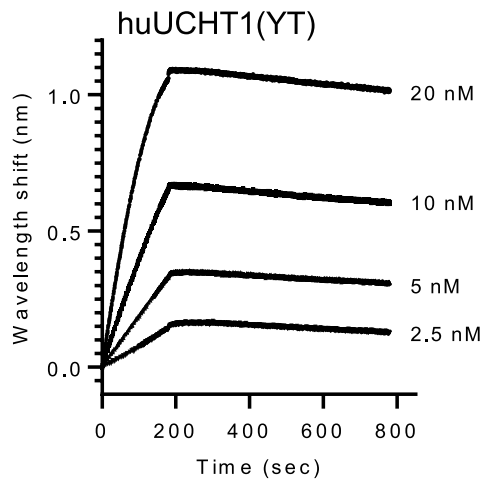

Figure 1. Optimized CD3 recruitment domain improves binding to $C D 3$ and augments surface expression. (A) An illustration of the receptor design. (B) MFI of receptor surface expression on transduced cells. Cells were gated on live $>$ singlets $>$ CD4/CD8 $>$ NGFR ${ }^{+}$. Data are representative of seven healthy donors. Statistical significance was determined using repeated measures ANOVA. (C) Binding of purified His-tagged UCHT1 variants to PBMCs. Flow cytometry data were gated on live $>$ singlets $>\mathrm{CD}^{+} / \mathrm{CD}^{+}$. MFIs of the protein stain were recorded at each protein concentration and converted to percent saturation based on the highest MFI value for each protein. (D) The impact of the Y-T mutation on the specific contacts formed between different UCHT1 variants and CD3E. Docking models created with ClusPro 2.0 software were analyzed for specific contacts with CSU software developed at the Weizmann Institute of Science. The total number of bonds predicted for each amino acid is shown. (E) Bio-layer interferometry comparison of humanized UCHT1 variants. Streptavidin probes were loaded with biotinylated human $\mathrm{CD} 3 \varepsilon \delta$ heterodimer and incubated with His-tagged, purified, humanized UCHT1 variants. Data were recorded using the Octet bio-layer interferometry system and analyzed with Prism 8. ANOVA, analysis of variance; Conc., concentration; CSU, contacts of structural units; MFI, median fluorescence intensity; sec, seconds.

${ }^{*} \mathrm{p} \leq 0.05$

analyzed with Living Image 4.2 software (PerkinElmer) for Mac OS X (Apple, Cupertino, CA, USA). Tumor burden was represented as the sum of the dorsal and ventral average radiance $\left(\mathrm{p} / \mathrm{s} / \mathrm{cm}^{2} /\right.$ sr) signal.

\section{Results}

\section{Optimized CD3-binding domain improves TAC T-cell efficacy}

TAC receptors lack synthetic signaling domains and activate $\mathrm{T}$ cells by co-opting the endogenous TCR-CD3 complex. Previously, the authors compared several CD3e-binding scFvs and described the rationale for choosing the UCHT1 domain for the TAC receptor scaffold (Figure 1A) [34]. Recognizing that the UCHT1 framework is of mouse origin and could give rise to human anti-mouse antibodies and potentially limit the clinical utility of TAC T cells, the authors replaced UCHT1 with a humanized version (huUCHT1) [47,48]. The authors recently identified a variant UCHT1 with a point mutation of $\mathrm{Y} \rightarrow \mathrm{T}$ at position 54, which improved TAC surface expression and growth of TAC T cells [49]. Given the potential benefits of the $\mathrm{Y} 54 \rightarrow \mathrm{T}$ mutation, in parallel, the authors also evaluated a variant of huUCHT1 with the Y54 $\rightarrow$ T mutation (huUCHT1[YT]). To focus on scaffold 
optimization, the authors used the same C11D5.3 BCMA-specific scFv as the antigen-binding domain in all scaffolds.

In all cultures, the CD4 T cells were 88.1-95.3\% transduced and the CD8 T cells were 83.6-93.8\% transduced based on expression of the transduction marker NGFR. The authors gated on the NGFR+ transduced cells to characterize receptor expression levels. The huUCHT1(YT) scaffold afforded higher TAC surface expression than the huUCHT1 scaffold in CD4 T cells, with a similar trend for higher expression in CD8 T cells (Figure 1B). At the protein level, both recombinant huUCHT1 and huUCHT1(YT) bound primary human T cells equally well and markedly better than UCHT1 (Figure 1C). Although in silico modeling suggested a potential decrease in huUCHT1(YT) affinity for CD3 (Figure 1D), bio-layer interferometry studies with purified huUCHT1 and huUCHT1(YT) showed that both proteins had high affinity for $\mathrm{CD} 3 \varepsilon \delta$, with a $\mathrm{K}_{\mathrm{d}}$ of approximately 100 pM (Figure 1E).

In vitro functional assessment revealed overall comparable postactivation cytokine expression profile across all three UCHT1 variants (Figure 2A; also see supplementary Figure 1A,B). UCHT1-TAC showed higher variation in cytokine production, compared to TACs with humanized UCHT1 scaffolds. The only statistically significant differences were observed in CD4 T cells, where huUCHT1(YT)-TAC produced more TNF- $\alpha$ than huUCHT1-TAC upon KMS-11 stimulation and UCHT1-TAC produced more TNF- $\alpha$ than huUCHT1-TAC upon MM.1S stimulation (Figure 2A; also see supplementary Figure B). All three constructs were equally efficient at killing KMS-11 and MM.1S tumors in vitro (Figure $2 \mathrm{~B}$; also see supplementary Figure $1 \mathrm{C}$ ). Irrespective of comparable in vitro performance, huUCHT1(YT)-TAC outperformed other constructs in vivo (Figure $2 \mathrm{C}$ ). Thus, considering the favorable surface expression profile and good in vitro and in vivo efficacy, the authors chose to move forward with the huUCHT1(YT)based scaffold.

Receptor surface expression differentially impacts early TAC T-cell activation

During the process of scaffold optimization, the authors observed that the CD8 $\alpha$ signal peptide consistently led to more BCMA-TAC on the cell surface of $\mathrm{T}$ cells from healthy donors compared with the IgK signal peptide (Figure 3A,B). A similar observation was made with $\mathrm{T}$ cells form MM patients (Figure $3 C$ ). The difference in surface expression was not due to a difference in vector copy number (see supplementary Table 1 ). As receptor density can affect performance of CAR $T$ cells [50-52], the authors investigated the effect of TAC receptor density on TAC T-cell performance. At baseline, surface expression of the TACs did not influence checkpoint receptors PD-1, TIM-3 and LAG-3 (see supplementary Figure 2A), which was consistent with the lack of tonic signaling produced by TAC receptors [34].

Receptor density should affect physical interactions between the TAC T cells and their target antigen. The authors compared the ability of TAC T cells to form conjugates with BCMA-positive KMS-11 cells (Figure 3D). Within the first $20 \mathrm{~min}, \mathrm{CD} 8 \alpha$ - and IgK-TAC T cells demonstrated equal ability to bind KMS-11 cells. However, by $40 \mathrm{~min}$, TAC T cells with lower receptor levels (IgK-TAC) started to disengage from the targets, whereas $\mathrm{CD} 8 \alpha$-TAC T cells continued binding more tumor cells. When MM.1S tumor targets were used, CD8 $\alpha$-TAC T cells were more efficient at binding tumor cells during earlier time points, with both types of T cells leveling off in their peak binding ability within 20 min (see supplementary Figure 2B). Similarly, when TAC T cells were exposed to a synthetic lipid bilayer functionalized with human BCMA and ICAM-1, CD $8 \alpha$-TAC T cells showed greater binding than IgK-TAC T cells (Figure $3 \mathrm{E}$ ). Taken together, these results indicated that higher levels of TAC receptor led to enhanced physical interaction between the TAC T cells and antigen-containing membranes.

The authors next sought to determine whether the enhanced physical associations mediated by the $\mathrm{CD} 8 \alpha$-TAC receptors would
A
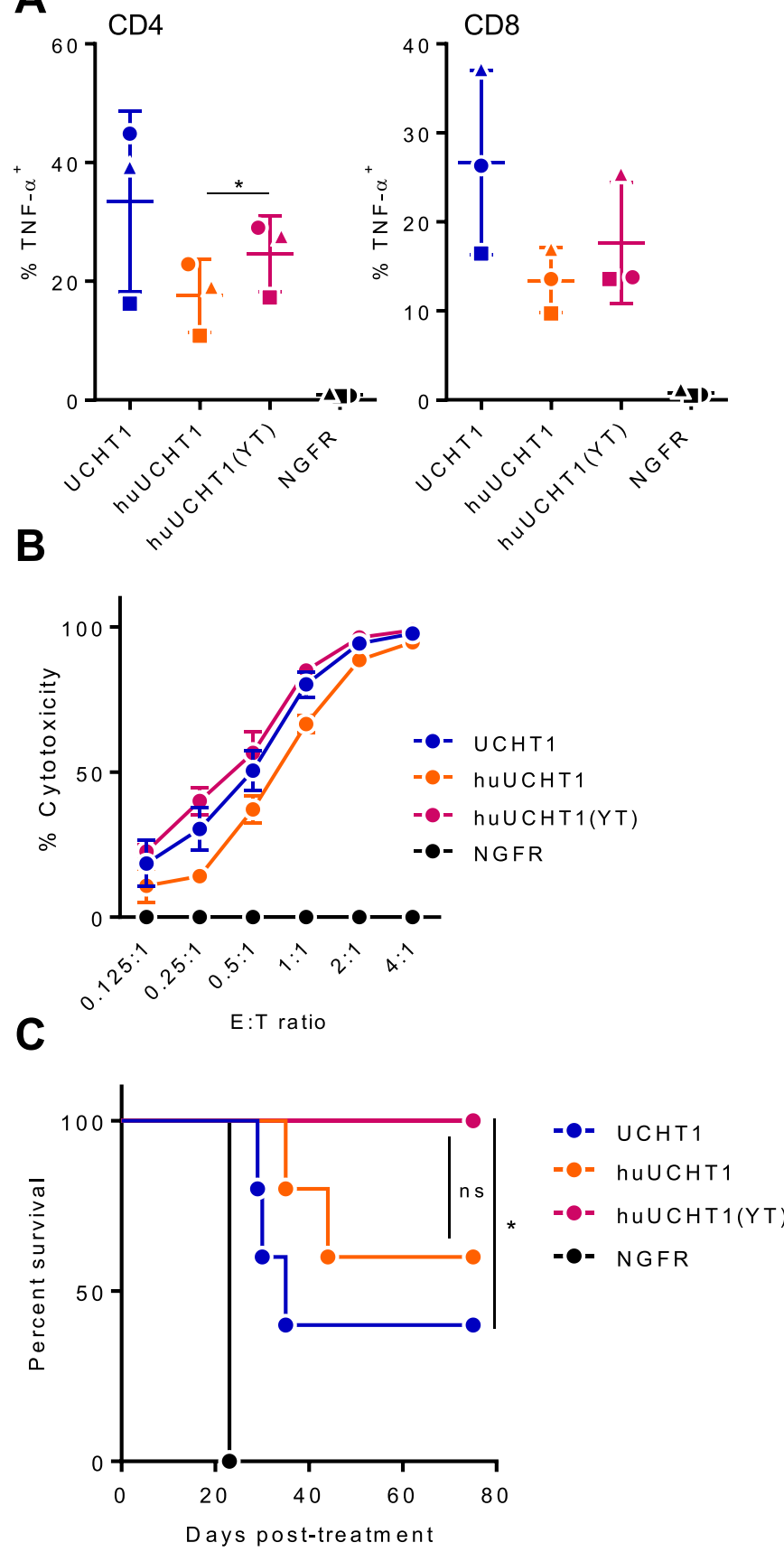

Figure 2. The huUCHT1(YT) variant outperforms other UCHT1 variants in vivo in the KMS-11 model. (A) Intracellular TNF- $\alpha$ expression. T cells were incubated with KMS11 tumor targets for $4 \mathrm{~h}$ at $37^{\circ} \mathrm{C}$ at a $2: 1 \mathrm{E}: \mathrm{T}$ ratio in the presence of brefeldin $\mathrm{A}$ and monensin and stained for surface CD4 and CD8 and intracellular IFN- $\gamma$, TNF- $\alpha$ and IL2. Plots were gated on live $>$ singlets $>$ CD4/CD $8>$ IFN $-\gamma$ versus TNF- $\alpha$. Statistical significance was determined with repeated measures ANOVA. Data from three healthy donors marked by different symbols are shown. Bars represent mean and SD. (B) In vitro cytotoxicity. T cells were incubated with effLuc-expressing KMS-11 tumor targets for $8 \mathrm{~h}$ at $37^{\circ} \mathrm{C}$ at different E:T ratios, and all conditions were repeated in triplicate. Luminescence was measured with an open filter upon addition of D-luciferin substrate $(0.15 \mathrm{mg} / \mathrm{mL})$. Mean and SD for each condition are displayed. Data are representative of four healthy donors. (C) Survival of KMS-11 tumor-bearing NRG mice treated with $3 \times 10^{6}$ cryopreserved TAC ${ }^{+}$T cells IV 12 days post-tumor injection. $\mathrm{N}=5$ mice per group. Statistical significance was determined with log-rank (Mantel-Cox) test. ANOVA, analysis of variance; effLuc, enhanced firefly luciferase; IV, intravenously; NRG, NOD.Cg-Rag1tm1MomIl2rgtm1Wjl/SzJ; SD, standard deviation.

ns $=$ non-significant $(\mathrm{p}>0.05)$.

${ }^{*} \mathrm{p} \leq 0.05$. 
A

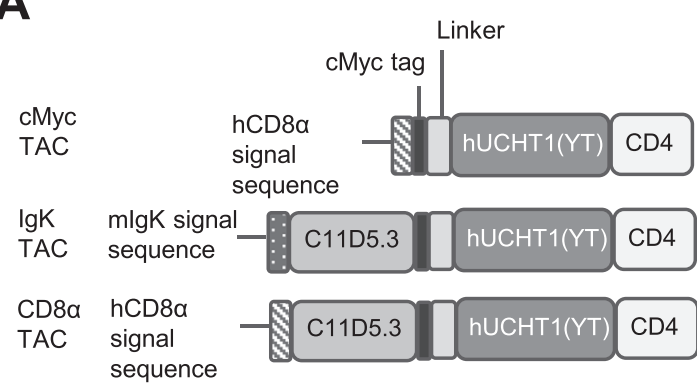

CD4

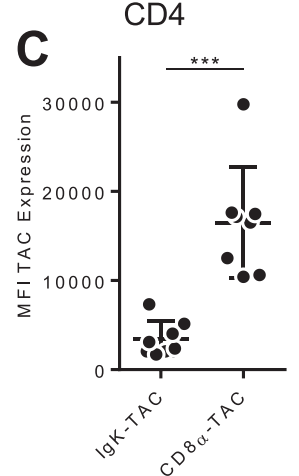

D

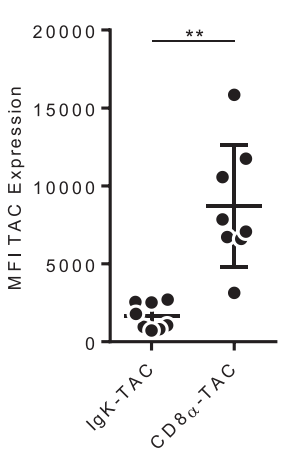

B

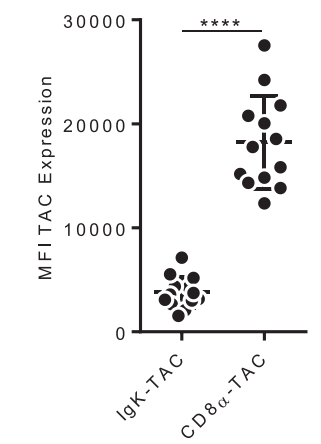

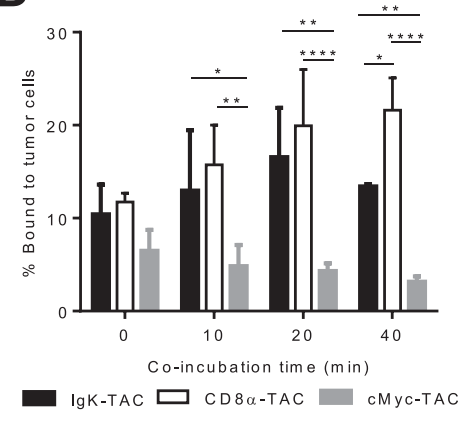

CD8
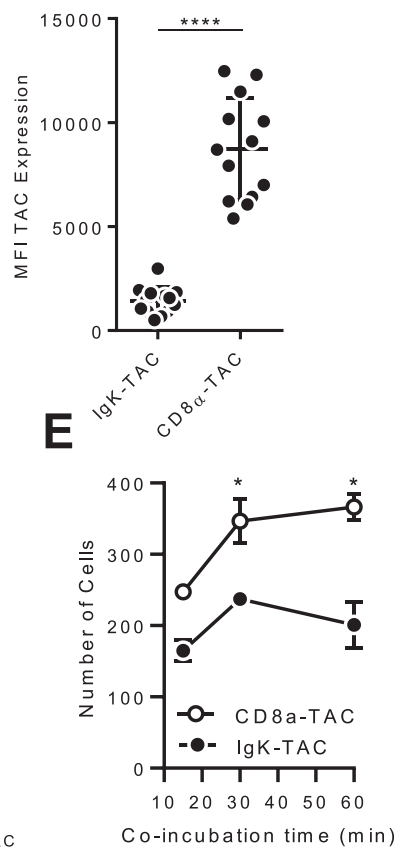

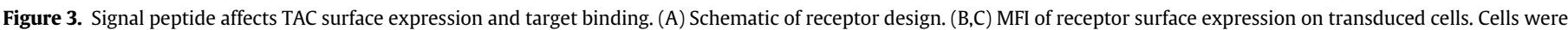

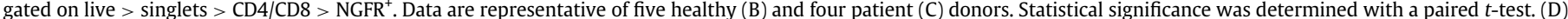

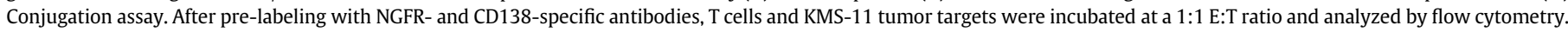

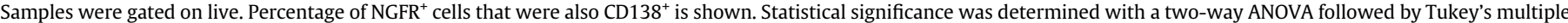

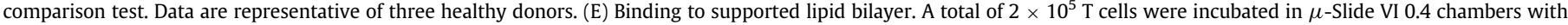

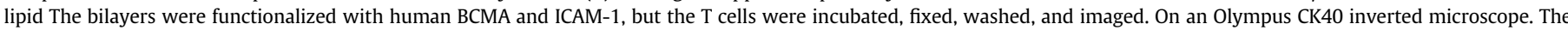

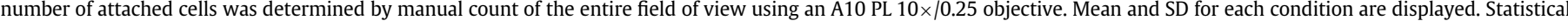

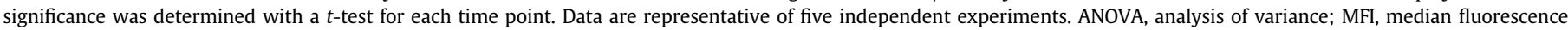
intensity; SD, standard deviation.

$$
\begin{aligned}
& { }^{*} \mathrm{p} \leq 0.05 \\
& { }_{* *} \mathrm{p} \leq 0.01 \\
& { }^{* * *} \mathrm{p} \leq 0.001 \\
& { }^{* * * *} \mathrm{p} \leq 0.0001
\end{aligned}
$$

translate into changes in immunological synapse formation. Both CD8 $\alpha$ - and IgK-TAC T cells formed a classical TCR-driven synapse (Figure 4A; also see supplementary Figure 4A) [53], characterized by a ring-like arrangement of F-actin and lymphocyte function-associated antigen 1 surrounding a central supramolecular activation cluster [54]. TAC T cells with higher receptor levels on the surface (CD8 $\alpha$ TAC) accumulated significantly more Lck in the synapse compared with TAC T cells with fewer receptors (IgK-TAC) (Figure 4B). This translated into faster polarization of perforin to the $\mathrm{T}$ cell-bilayer interface in CD8 $\alpha$-TAC T cells, with IgK-TAC T cells catching up within the first hour of activation (Figure 4C). By $30 \mathrm{~min}$, both CD $8 \alpha$ - and IgK-TAC T cells had polarized equivalent amounts of perforin (Figure 4D).

Despite differences in early activation, TAC T cells with different receptor levels are equally efficacious in vitro

Given the more rapid maturation of the synapse in CD8 $\alpha$-TAC T cells, the authors asked whether this would influence TCR-dependent signaling. To address this question, the authors measured CD69 (surrogate for T-cell activation) and Nur77 (surrogate for T-cell signal strength) [55] on the TAC T cells stimulated with KMS-11 cells. At the 2-h time point, there was a trend for more Nur77 activation in CD8 $\alpha$ TAC T cells relative to IgK-TAC T cells, but this was not observed in all T-cell batches (Figure 4E,F; also see supplementary Figure 3B). The authors questioned whether higher receptor levels could lead to overstimulation of TAC T cells and higher levels of activation-induced cell death. Measurement of cleaved caspase-3 following stimulation of $\mathrm{CD} 8 \alpha$ - and IgK-TAC T cells revealed no differences in cell death post-stimulation (see supplementary Figure 3C). Examination of the expression of activation marker CD69 and checkpoint receptors PD1,TIM-3 and LAG-3 across a broad time frame (4-96 h) following stimulation also demonstrated no difference in levels or kinetics (see supplementary Figure 4).

The results with Nur77 and CD69 suggested that the TAC T cells were activated equally at both receptor densities. Using healthy donor-derived TAC T cells, the authors noted that increased TAC expression had no effect on production of IFN- $\gamma$ and TNF- $\alpha$ following stimulation with KMS-11 cells (Figure 5A). However, more TAC receptors on the T-cell surface led to significantly improved IL-2 production by $\mathrm{CD} 4^{+} \mathrm{TAC} \mathrm{T}$ cells and a similar trend in $\mathrm{CD}^{+} \mathrm{T}$ cells. Comparable results were obtained with MM.1S targets (see supplementary Figure 5A,B). Receptor density had a more striking impact on early cytokine production in TAC T cells generated from myeloma patients. Higher receptor levels led to significantly more CD4 and CD8 TAC T cells producing IFN- $\gamma$ and TNF- $\alpha$ following 4-h stimulation with KMS-11 myeloma cells (Figure 5B). Similar results were obtained when MM.1S tumor targets were used for stimulation (see supplementary Figure $5 \mathrm{C}$ ). Nevertheless, by $24 \mathrm{~h}$ of stimulation, both IgK- and CD $8 \alpha$-TAC T cells produced equivalent levels of cytokines regardless of whether the $\mathrm{T}$ cells were obtained from healthy donors or MM patients (see supplementary Figure 6).

Since IL- 2 was elevated in CD8 $\alpha$-TAC T cells earlier than in IgKTAC T cells and this cytokine is known to support T-cell proliferation upon activation [56], the authors measured proliferative capacity of the BCMA-TAC T cells. In a reductionist system in which TAC T cells 
A

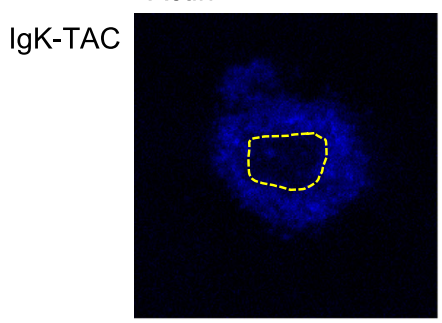

CD8 $\alpha-T A C$

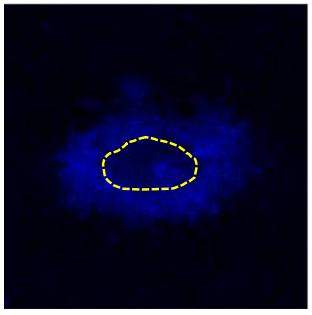

B

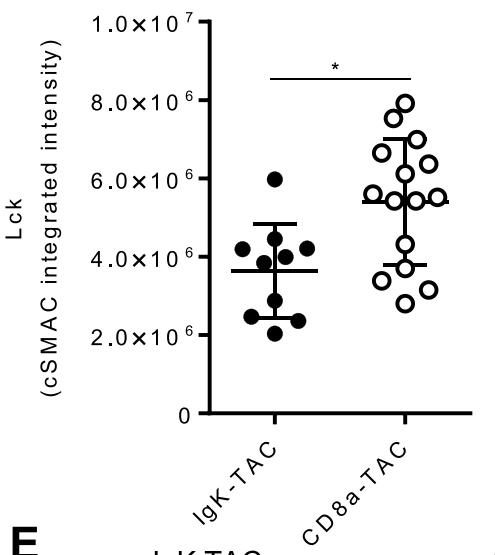

$\mathbf{E}$

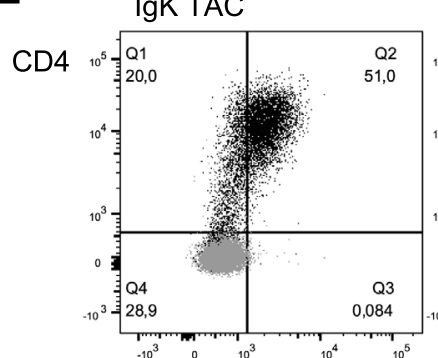

CD8 $\alpha$ TAC
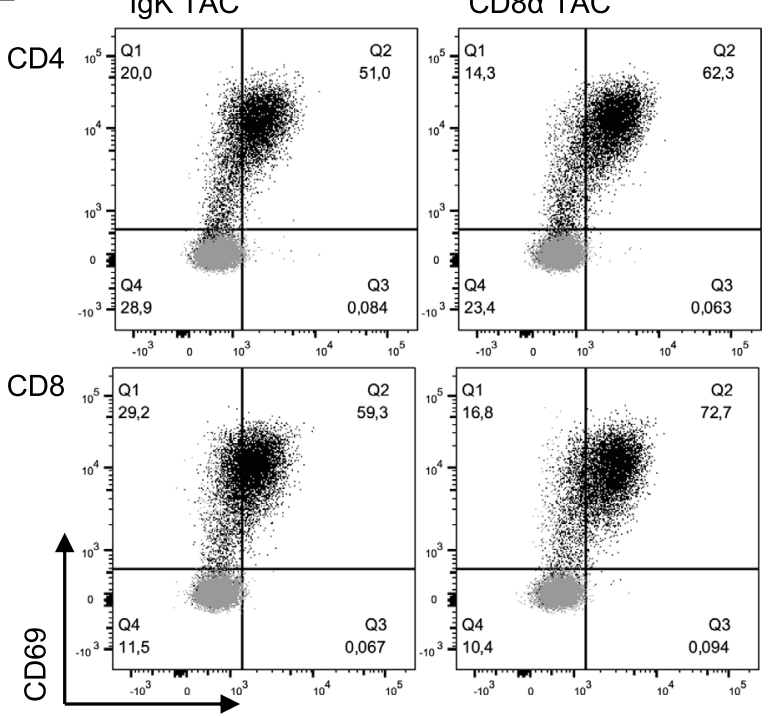

Nur77

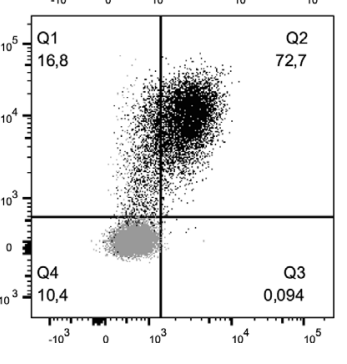

Lck
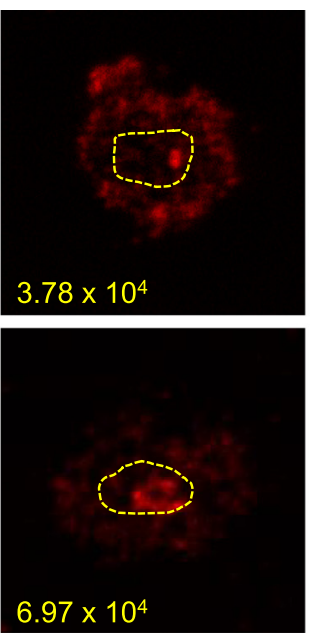

C

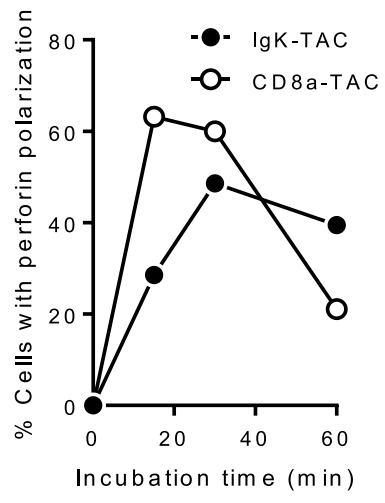

$\mathbf{F}$
Composite
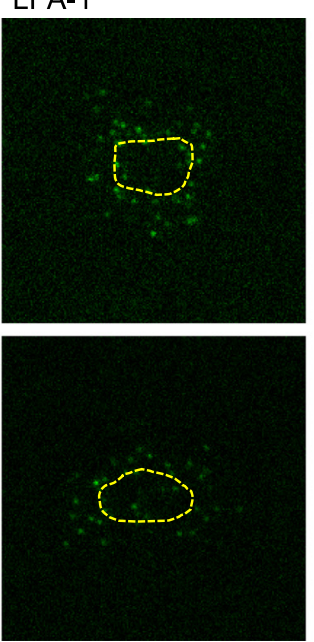

D
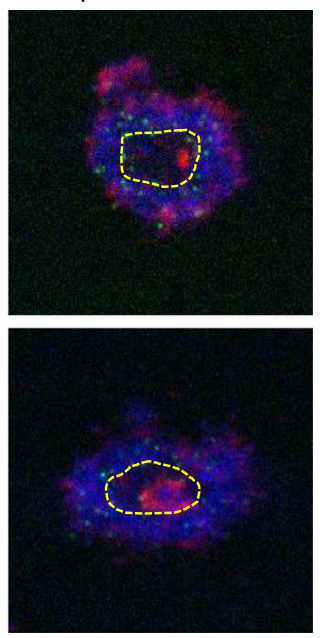

D
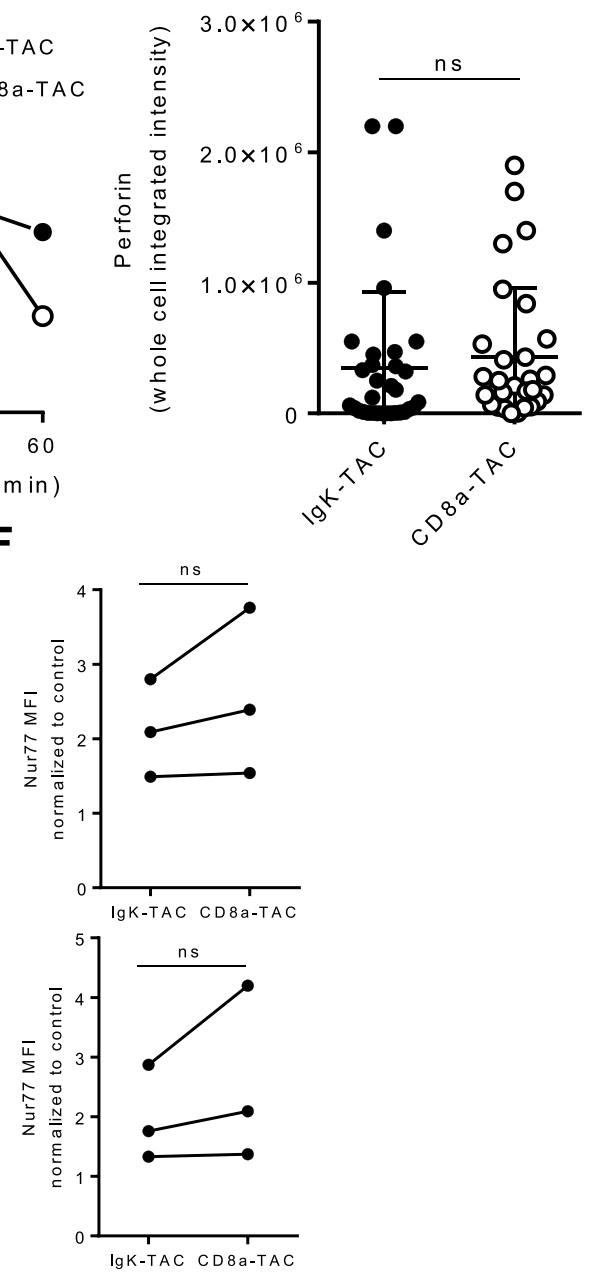

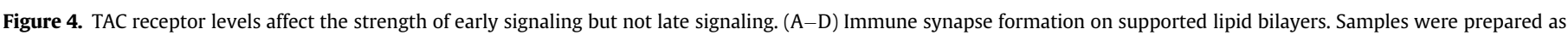

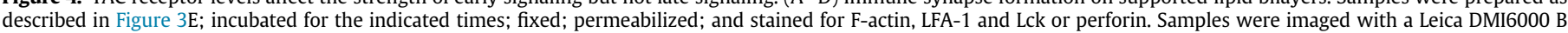

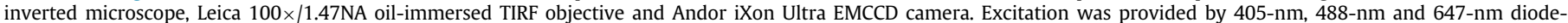

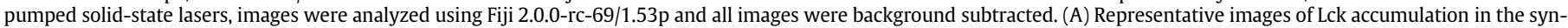

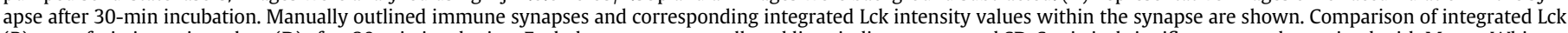

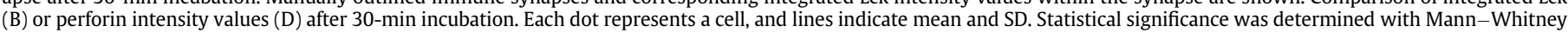

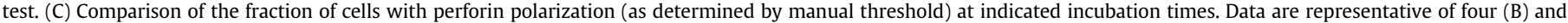

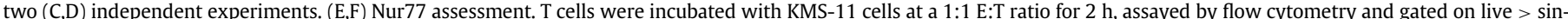

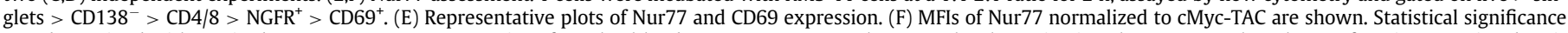

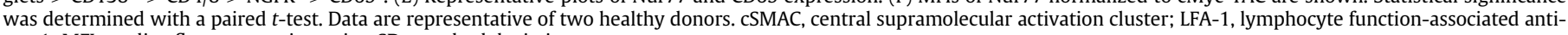
gen 1; MFI, median fluorescence intensity; SD, standard deviation.

$\mathrm{ns}=$ non-significant $(\mathrm{p}>0.05)$

$* \mathrm{p} \leq 0.05$. 
A

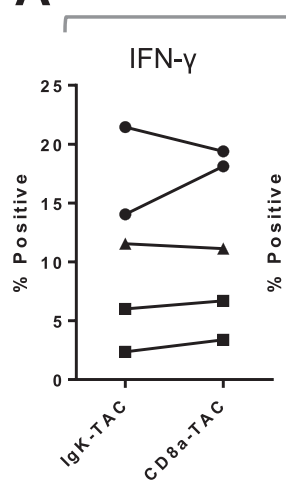

B

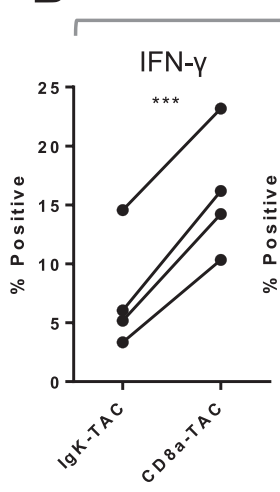

CD4

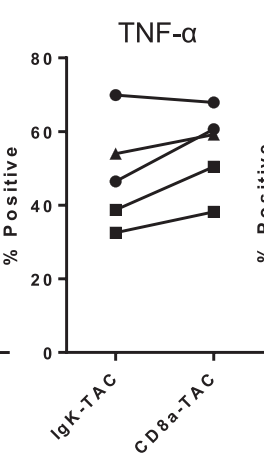

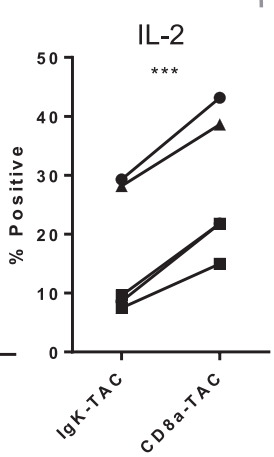
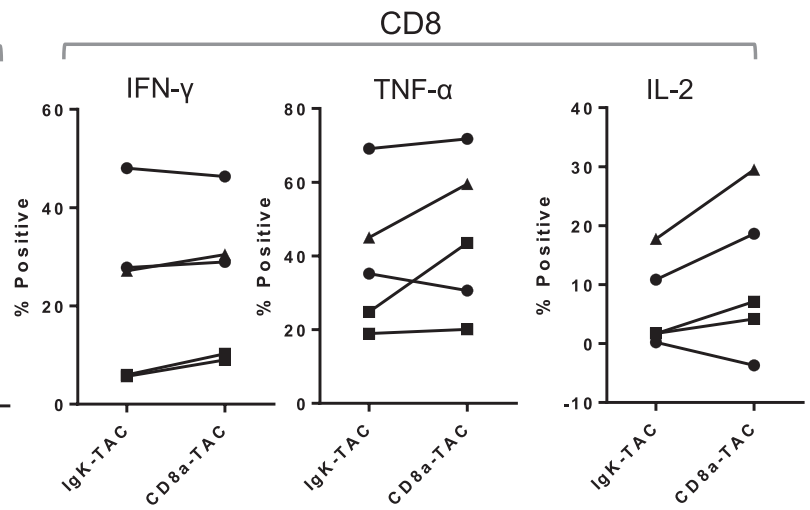

CD4
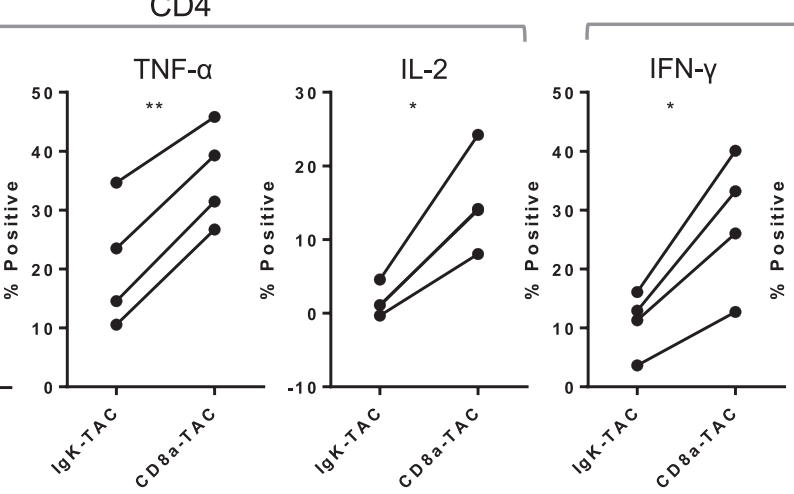

CD8
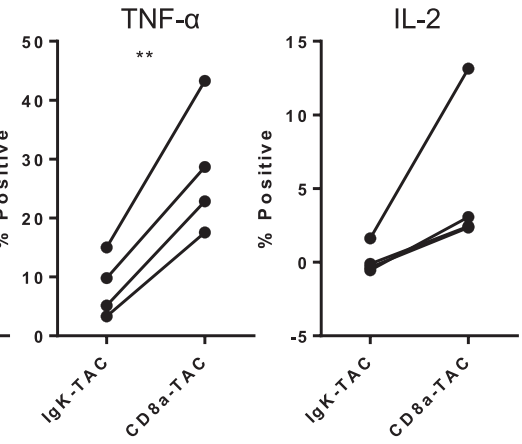

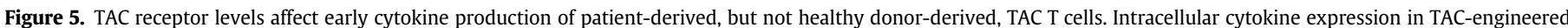

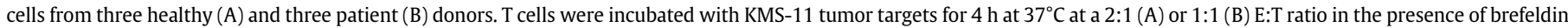

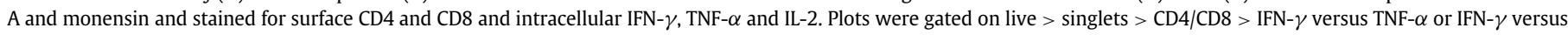
IL-2. Statistical significance was determined with a paired $t$-test.

${ }^{*} \mathrm{p} \leq 0.05$.

** $\mathrm{p} \leq 0.01$

were stimulated with BCMA-Fc protein-coated polystyrene beads, TAC T cells with higher receptor levels ( $C D 8 \alpha-\mathrm{TAC})$ were more proliferative over the range of antigen densities tested (Figure 6A,B). However, when stimulated with BCMA-positive KMS-11 (Figure 6C) and MM.1S (see supplementary Figure 5D) cells, both types of TAC T cells proliferated equally well, suggesting that additional cell-cell contacts mediated by cell adhesion molecules may mitigate the effects of differential TAC receptor surface levels. Interestingly, proliferation of patient-derived CD4 TAC T cells was reduced by high surface expression of the receptor (Figure 6E). Irrespective of the donor source (healthy or patient) and tumor target (KMS-11 or MM.1S), TAC receptor density had no impact on cytotoxicity, as both IgK- and CD8 $\alpha$ BCMA-TAC $T$ cells efficiently eliminated tumor cells in vitro (Figure 6D; also see supplementary Figure 5E).

\section{TAC T cells show robust in vivo efficacy across different receptor levels}

Receptor expression-dependent differences at the cell-cell binding and early signaling levels did not translate into marked functional differences in vitro. To assess whether therapeutic outcomes would be affected by receptor expression levels, the authors evaluated antitumor efficacy using an orthotopic, disseminated KMS-11 xenograft model. Using T cells from healthy donors, a single dose of $2 \times 10^{6}$ $\mathrm{CD} 8 \alpha$ - or IgK-TAC T cells per mouse was equally effective at clearing tumors and inducing sustained remissions (Figure 7A; also see supplementary Figure 7A). Titrating down the dose of T cells to suboptimal therapeutic levels also did not reveal statistically significant differences between TAC T cells with higher and lower receptor levels (Figure 7A; also see supplementary Figure 7B). Similarly, the therapeutic efficacy of MM patient-derived TAC $T$ cells was not

significantly affected by the differences in TAC receptor density over a range of TAC T-cell doses (Figure $7 \mathrm{~B}$; also see supplementary Figure 7C,D). The authors also tested the two TAC T-cell populations in the MM.1S model and again found no difference in therapeutic outcomes (see supplementary Figure 8). Thus, despite the influence of TAC receptor density on early events following TAC engagement (cell binding, Lck recruitment, perforin polarization), receptor density did not impact therapeutic efficacy.

\section{Discussion}

The robust therapeutic activity of CAR T cells has prompted research into other receptor designs that retain the therapeutic benefit of current-generation CAR T cells but diminish the unwanted toxicity profile. Recently, several alternate synthetic antigen receptors have been described that focus on co-opting natural TCR signaling, including the authors' TAC platform [34,57,58]. In these studies, TCR-centric receptors were equally or more efficacious than conventional second-generation CARs and less likely to produce cytokines in vitro or cause in vivo toxicities. These observations suggest an improved ability of $T$ cells engineered with these receptors to mediate effector responses. Unlike other TCR-centric synthetic receptors, TAC does not replace either CD3 or TCR chains. Rather, the TAC receptor redirects endogenous receptor complex through non-covalent association with the $\mathrm{CD} 3 \varepsilon$ chain. Here the authors described the next generation of the TAC platform. These studies focused on the development of a BCMA-specific TAC receptor, but the optimized TAC scaffold described herein should be broadly useful against any tumor target.

For backbone optimization, the authors designed all receptors with the same antigen-binding domain. The authors selected the 


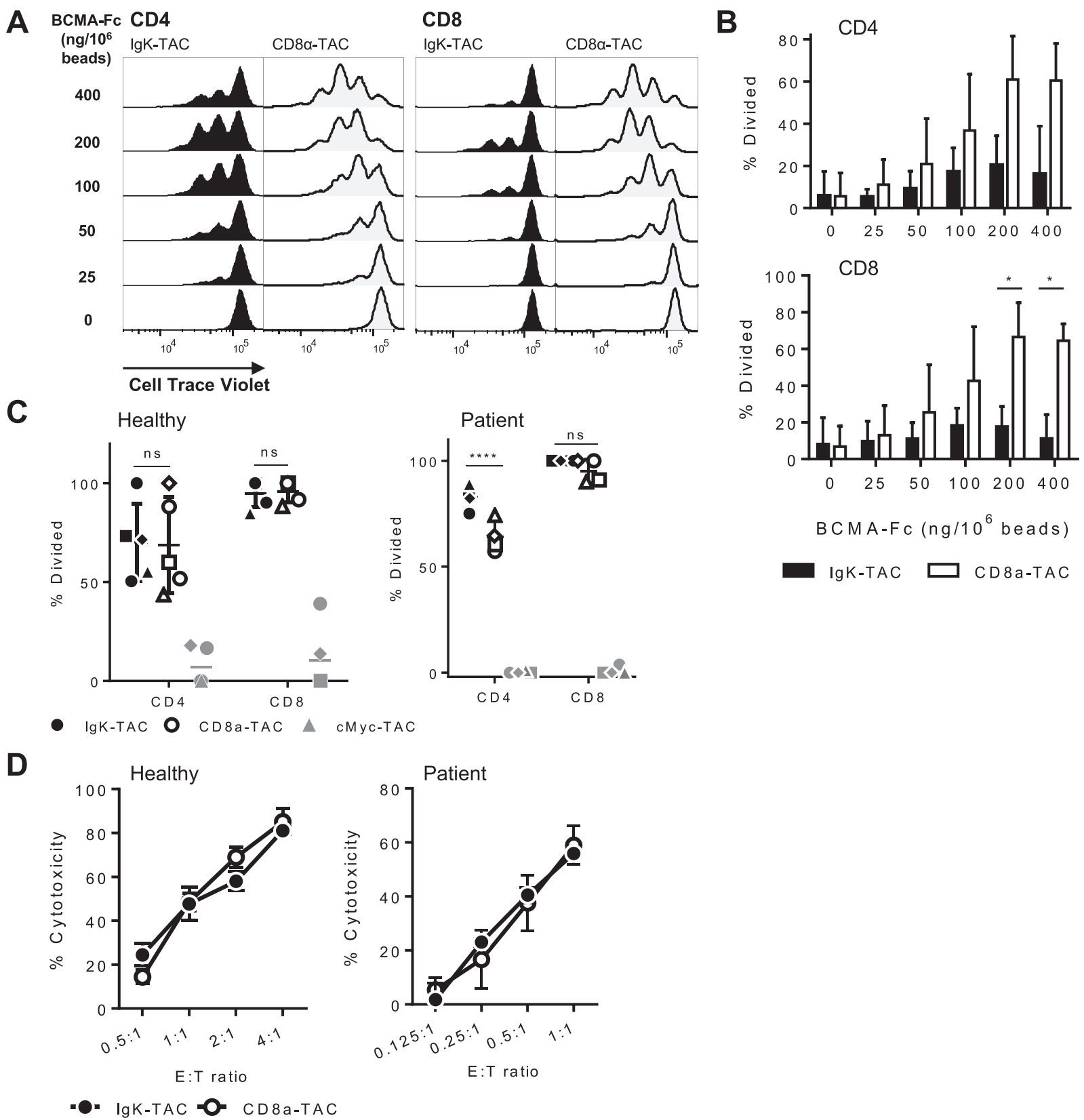

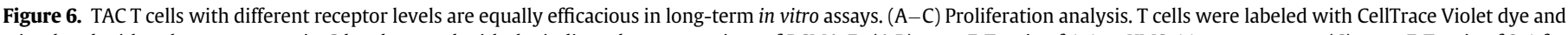

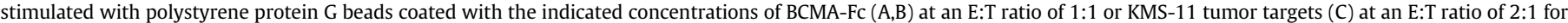

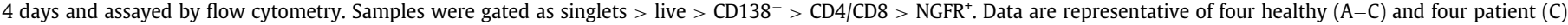

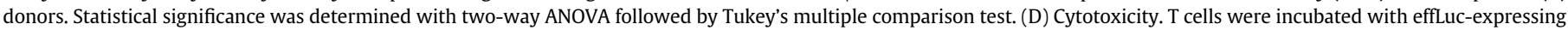

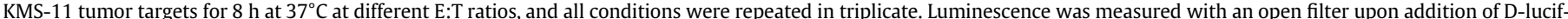

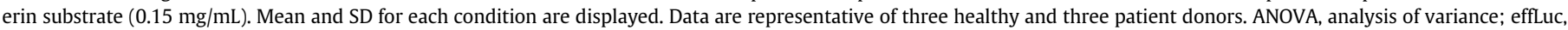
enhanced firefly luciferase; SD, standard deviation.

ns $=$ non-significant $(\mathrm{p}>0.05)$

* $\mathrm{p} \leq 0.05$.

$* * * * \mathrm{p} \leq 0.0001$.

C11D5.3 scFv for these studies, as this scFv has been clinically validated on a CAR scaffold [16]. The authors' in vitro optimization of the TAC scaffold revealed that humanization of the UCHT1 domain improved binding to CD3 on the surface of T cells. Substitution of huUCHT1 with a version carrying the Y54 $\rightarrow$ T mutation (huUCHT1 [YT]) further improved surface expression and in vivo efficacy. In the authors' original description of the TAC, UCHT1 emerged as the most effective CD3 binder of the five scFvs compared [34]. Here the subtle changes to UCHT1 (humanization and single point mutation) further improved the TAC receptor performance, underscoring the importance of the TAC-CD3 interaction. Despite the in silico- predicted reduction in the number of bonds between UCHT1 and $\mathrm{CD} 3 \varepsilon$, the $\mathrm{Y} 54 \rightarrow \mathrm{T}$ mutation did not impact binding of recombinant scFvs to human $T$ cells, nor did it affect affinity. The improved performance of the huUCHT1(YT) variant likely reflects subtle changes in the protein-protein interactions between UCHT1 and CD3, which are more complicated than simple binding strength and are the subject of further investigation. Based on the results described herein, the authors elected to move forward with huUCHT1(YT) as our optimized TAC scaffold for clinical testing and are currently screening humanized BCMA-specific scFvs to generate a fully humanized TAC for MM. 


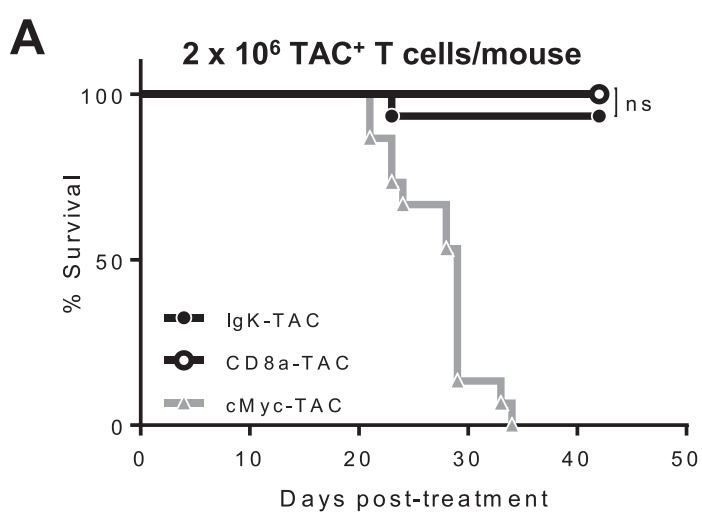

B

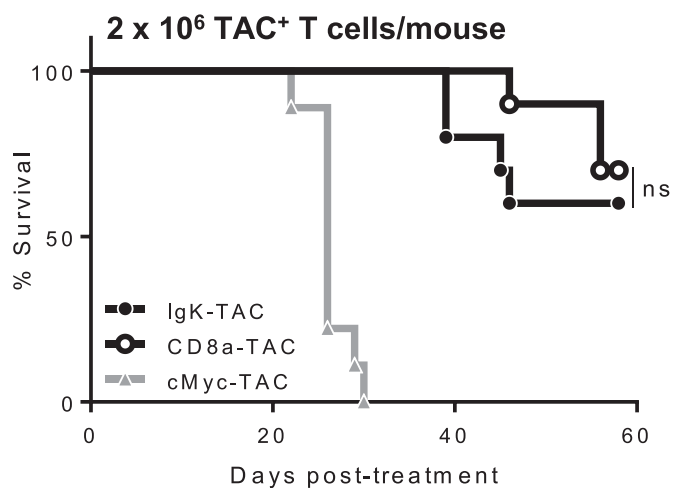

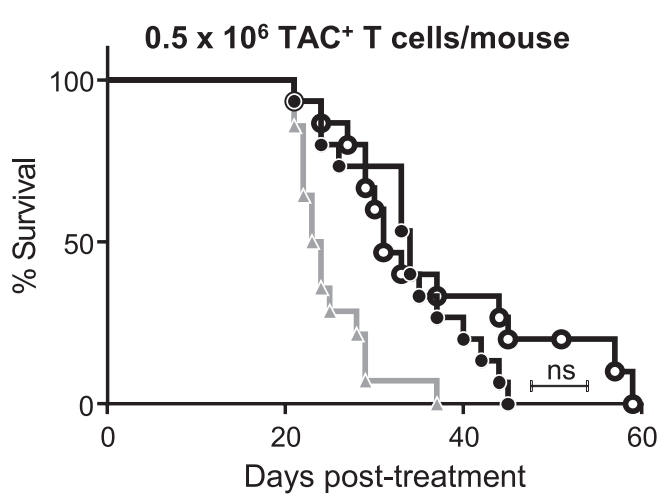

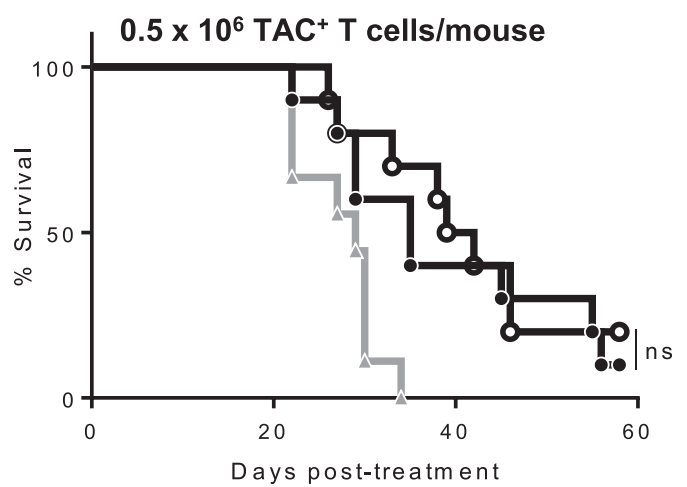

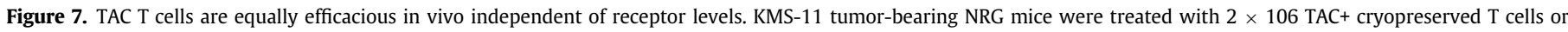

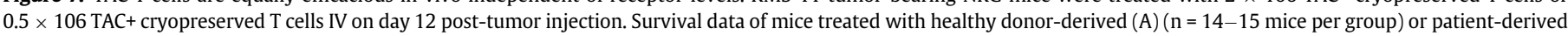

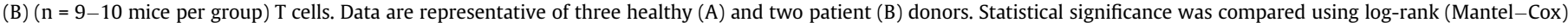
test. IV, intravenously; NRG, NOD.Cg-Rag1tm1MomIl2rgtm1Wjl/SzJ.

$\mathrm{ns}=$ non-significant $(\mathrm{p}>0.05)$.

The authors' observation of the signal peptide impacting receptor expression levels created an interesting system for evaluating the relationship between TAC surface levels and TAC T-cell functionality. The signal peptide targets proteins destined for secretion or surface expression to the endoplasmic reticulum (ER) and gets cleaved from the newly synthesized polypeptide chain upon translocation to the ER [59]. Length and amino acid composition of signal peptides affect kinetics and efficiency of binding to the signal recognition particle, translocation into the ER or cleavage by the signal peptide peptidase [60]. Thus, by using different signal peptides, the authors likely affected one or more of these processing steps of the TAC receptor synthesis.

The CD28 co-stimulatory domain in second-generation CARs mediates tonic signaling during the manufacturing period, which can produce exhaustion of the engineered T-cell product [50,61]. Consequently, some of the strategies to reduce tonic signaling have focused on limiting CAR surface expression by optimizing promoter activity [50-52,62] or using logic-gated receptors [63]. Here the authors show that TAC T cells do not upregulate exhaustion markers, irrespective of TAC receptor expression levels. These observations support the authors' rationale for excluding endogenous signaling domains from the TAC scaffold in favor of allowing TAC-engineered cells to auto-regulate through the endogenous TCR-dependent pathways.

The experiments with supported lipid bilayers functionalized with BCMA antigen and ICAM-1 improved the authors' understanding of the immunological synapse formed by TAC T cells. The structure of TAC receptor-driven synapses was similar to the conventional bullseye structure of the TCR synapse [64], suggesting that TACs function as the authors intended, by redirecting endogenous TCRs to the target of interest. Higher TAC surface levels improved target binding and increased Lck accumulation at the immunological synapse.
Consistent with the role of Lck in driving lytic granule polarization and degranulation [65], the authors observed that TAC T cells with higher TAC receptor levels displayed more rapid perforin polarization. Nevertheless, similar levels of perforin polarization and equivalent cytotoxicity against tumor cells were ultimately achieved independent of TAC receptor expression. Given the observation that TAC receptor expression did not influence the strength of TCR signaling (measured indirectly by Nur77 expression) and activationinduced cell death, the authors believe that although the different levels of TAC expression may influence upstream features of T-cell activation (i.e., target binding, recruitment of Lck to the immunological synapse), these events do not ultimately influence the activation threshold of the T cell or impact therapeutic efficacy.

In a reductionist bead-based activation system where only receptor-ligand interactions were present, higher TAC surface levels reduced the amount of antigen needed to trigger proliferation of TAC $\mathrm{T}$ cells. These results are consistent with previous work linking proliferation with TCR surface density when antigen concentration was low [66]. In cell-based stimulation, receptor-ligand interactions were likely augmented by contacts between cell adhesion molecules. For example, integrin lymphocyte function-associated antigen 1 expressed on T cells lowers the threshold for T-cell activation by triggering the Erk1/2 pathway and mediating activator protein 1 activity [67]. The additional cell-cell contacts achieved through surface ligands on the tumor cells enhanced TAC T-cell proliferation compared with antigen-coated beads, indicating that the magnitude of TAC expression is not relevant to proliferative responses when ancillary contacts are available.

The authors noted that T-cell effector functions were differentially susceptible to TAC receptor expression levels. The authors' data from healthy donor-derived TAC T cells suggest that IL-2 production is more sensitive to receptor surface expression than TNF- $\alpha$ or IFN- $\gamma$ 
production. These results mirror findings from prior studies in T-cell biology. During chronic viral infection, the ability to produce IL-2 is lost before the ability to produce IFN- $\gamma$ and TNF- $\alpha$ [68]. Coincidentally, when TCR ligand concentration is limited, IL-2 production is hindered before IFN- $\gamma$ production [69]. Taken together, these data and the authors' observations support higher activation threshold requirements for IL-2 production.

Interestingly, in patient-derived TAC T cells, all three of the effector cytokines tested were affected by surface levels of the receptor. MM patient-derived T cells can downregulate CD28 [70], phosphoinositide 3-kinase, $\mathrm{CD} 3 \zeta$ and Lck [71], although reports on some of the signaling mediators are inconsistent [72]. Inadequate availability of signaling molecules downstream of TCR activation could elevate the activation threshold for patient-derived versus healthy $T$ cells, which offers an explanation for the increased sensitivity of cytokine secretion to TAC receptor levels when patient cells were used. The receptor expression-dependent differences in early cytokine production observed in a short-term intracellular cytokine staining assay were not detected in longer-term assays (proliferation, overnight cytotoxicity and cytokine accumulation) in healthy or MM patient-derived T cells. Thus, although the receptor expression levels may impact early events post-antigen engagement, expression levels do not impact later events, which are likely more pertinent to therapeutic efficacy.

The engineered T-cell product ultimately relies on a combination of effector functions to mount an anti-tumor response in vivo. Thus, it was important to test whether any of the differences the authors observed in vitro would influence efficacy in animal models of MM using TAC T cells from healthy and patient donors. Equivalent efficacy in vivo from TAC T-cell products carrying different levels of TAC receptor highlighted the robustness of the TAC platform and its stable performance irrespective of receptor expression levels. Interestingly, although the original UCHT1 and next-generation huUCHT1(YT) scaffolds displayed similar in vitro functionality, huUCHT1(YT)-TAC T cells were more efficacious in vivo. This observation highlights the limitation of in vitro systems for comparing cell-based therapies and argues for the necessity of in vivo testing.

\section{Conclusions}

In this study, the authors have described further evolution of the TAC scaffold that yields a TAC T-cell product that is efficacious against xenograft mouse models of MM. The authors conducted a detailed assessment of different BCMA-TAC T-cell functions and characterized how these functions were affected by changes in receptor surface expression. The authors have also tested this scaffold in other models of hematologic malignancy with comparable robust therapeutic outcomes. Ultimately, the authors established that TAC T cells are equally efficacious across a range of receptor levels. This work serves as a basis for the authors' future modifications to the TAC T-cell platform and brings us closer to testing BCMA-TAC T cells clinically.

\section{Declaration of Competing Interest}

JLB has an ownership interest in and receives research funding from Triumvira Immunologics Inc. KB and GD are co-inventors on a patent related to TAC receptors. JLB is a co-inventor on several patents related to TAC receptors and oncolytic viruses.

\section{Funding}

This research was funded by support from the Samuel Family Foundation and BioCanRx. KB received support from the Ontario Graduate Scholarship. MS received support from the Natural Sciences and Engineering Research Council of Canada Undergraduate Student Research Award program. JLB was supported by the Canadian
Research Chair in Translational Immunology and the John Bienenstock Chair in Molecular Medicine.

\section{Author Contributions}

K.B. and J.L.B. designed the experiments. K.B., D.C., G.D., and M.S. performed molecular and cellular work. G.D. performed in silico modeling. J.D.M. performed multi-parametric analysis of the checkpoint receptor expression. R.C.T. and A.F.R. worked on protein purification and bio-layer interferometry analysis. D.M. conducted microscopy studies. K.B., C.L.B., and C.A. performed in vivo studies. S. R.F. facilitated patient sample collection. K.B. and J.L.B. interpreted experimental results and wrote the manuscript. All authors read and approved the manuscript.

\section{Data Availability}

Data and materials are available from the authors under a data or material transfer agreement.

\section{Supplementary materials}

Supplementary material associated with this article can be found in the online version at doi:10.1016/j.jcyt.2021.05.007.

\section{References}

[1] Holstein SA, McCarthy PL. Immunomodulatory Drugs in Multiple Myeloma: Mechanisms of Action and Clinical Experience. Drugs 2017;77(5):505-20.

[2] Asatsuma-Okumura T, Ito T, Handa H. Molecular mechanisms of cereblon-based drugs. Pharmacol Ther 2019;202:132-9.

[3] Kunacheewa C, Orlowski RZ. New Drugs in Multiple Myeloma. Annu Rev Med 2019;70:521-47.01

[4] Ghobrial I, Cruz CH, Garfall A, et al. Immunotherapy in Multiple Myeloma: Accelerating on the Path to the Patient. Clin Lymphoma Myeloma Leuk 2019;19 (6):332-44

[5] Kriegsmann K, Kriegsmann M, Cremer M, et al. Cell-based immunotherapy approaches for multiple myeloma. Br J Cancer 2019;120(1):38-44. 01.

[6] Yu B, Liu D. Antibody-drug conjugates in clinical trials for lymphoid malignancies and multiple myeloma. J Hematol Oncol 2019;12(1):94.

[7] Iftikhar A, Hassan $\mathrm{H}$, Iftikhar N, et al. Investigational Monoclonal Antibodies in the Treatment of Multiple Myeloma: A Systematic Review of Agents under Clinical Development. Antibodies (Basel) 2019;8(2):34.

[8] Hatzoglou A, Roussel J, Bourgeade MF, et al. TNF receptor family member BCMA (B cell maturation) associates with TNF receptor-associated factor (TRAF) 1, TRAF2, and TRAF3 and activates NF-kappa B, elk-1, c-Jun N-terminal kinase, and p38 mitogen-activated protein kinase. J Immunol 2000;165(3):1322-30.

[9] O'Connor BP, Raman VS, Erickson LD, et al. BCMA is essential for the survival of long-lived bone marrow plasma cells. J Exp Med 2004;199(1):91-8.

[10] Novak AJ, Darce JR, Arendt BK, et al. Expression of BCMA, TACI, and BAFF-R in multiple myeloma: a mechanism for growth and survival. Blood 2004;103 (2):689-94

[11] Carpenter RO, Evbuomwan MO, Pittaluga S, et al. B-cell maturation antigen is a promising target for adoptive T-cell therapy of multiple myeloma. Clin Cancer Res. 2013;19(8):2048-60

[12] Trudel S, Lendvai N, Popat R, et al. Antibody-drug conjugate, GSK2857916, in relapsed/refractory multiple myeloma: an update on safety and efficacy from dose expansion phase I study. Blood Cancer J. 2019;9(4):37. 03.

[13] Topp MS, Duell J, Zugmaier G, et al. Evaluation of AMG 420, an anti-BCMA bispecific T-cell engaged (BiTE) immunotherapy, in R/R multiple myeloma (MM) patients: Updated results of a first in-human $(\mathrm{FIH})$ phase I dose escalation study. presented at: ASCO Annual Meeting; 2019; Journal of Clinical Oncology 37, no. 15_suppl (May 20, 2019) 8007-8007. https://doi.org/10.1200/JCO.2019.37.15_suppl.8007. https://ascopubs.org/doi/abs/10.1200/JCO.2019.37.15_suppl.8007

[14] Cohen AD, Melenhorst JJ, Garfall AL, et al. Predictors of T Cell Expansion and Clinical Responses Following B-Cell Maturation Antigen-Specific Chimeric Antigen Receptor T Cell Therapy (CART-BCMA) for Relapsed/Refractory Multiple Myeloma (MM). Blood 2018;132(Supplement 1). 1974-1974.

[15] Brudno JN, Maric I, Hartman SD, et al. T Cells Genetically Modified to Express an Anti-B-Cell Maturation Antigen Chimeric Antigen Receptor Cause Remissions of Poor-Prognosis Relapsed Multiple Myeloma. J Clin Oncol 2018;36(22):2267-80. 08.

[16] Raje N, Berdeja J, Lin Y, et al. Anti-BCMA CAR T-Cell Therapy bb2121 in Relapsed or Refractory Multiple Myeloma. N Engl J Med. 2019;380(18):1726-37. 05.

[17] Xu J, Chen LJ, Yang SS, et al. Exploratory trial of a biepitopic CAR T-targeting B cell maturation antigen in relapsed/refractory multiple myeloma. Proc Natl Acad Sci U S A 2019;116(19):9543-51. 
[18] Berdeja JG, Alsina M, Shah ND, et al. Updated Results from an Ongoing Phase 1 Clinical Study of bb21217 Anti-Bcma CAR T Cell Therapy. Blood 2019;134(Supplement_1). 927-927.

[19] Costello CL, Gregory TK, Ali SA, et al. Phase 2 Study of the Response and Safety of P-Bcma-101 CAR-T Cells in Patients with Relapsed/Refractory (r/r) Multiple Myeloma (MM) (PRIME). Blood 2019;134(Supplement_1). 3184-3184.

[20] Madduri D, Usmani SZ, Jagannath S, et al. Results from CARTITUDE-1: A Phase 1b/ 2 Study of JNJ-4528, a CAR-T Cell Therapy Directed Against B-Cell Maturation Antigen (BCMA), in Patients with Relapsed and/or Refractory Multiple Myeloma (R/R MM). Blood 2019;134(Supplement_1). 577-577.

[21] Mailankody S, Htut M, Lee KP, et al. JCARH125, Anti-BCMA CAR T-cell Therapy for Relapsed/Refractory Multiple Myeloma: Initial Proof of Concept Results from a Phase 1/2 Multicenter Study (EVOLVE). Blood 2018;132(Supplement 1). 957-957.

[22] Cohen AD, Garfall AL, Stadtmauer EA, et al. B cell maturation antigen-specific CAR T cells are clinically active in multiple myeloma. J Clin Invest 2019;129(6):2210-21. 03.

[23] Han L, Gao Q, Zhou K, et al. The phase I clinical study of CART targeting BCMA with humanized alpaca-derived single-domain antibody as antigen recognition domain. Journal of Clinical Oncology 2019;37(15_suppl). 2535-2535.

[24] Khalaf WS, Garg M, Mohamed YS, Stover CM, Browning MJ. Generation of Cytotoxic T Cells With Potential for Adoptive Tumor Immunotherapy of Multiple Myeloma. Front Immunol 2019;10:1792.

[25] Stadtmauer EA, Faitg TH, Lowther DE, et al. Long-term safety and activity of NYESO-1 SPEAR T cells after autologous stem cell transplant for myeloma. Blood Adv 2019;3(13):2022-34.

[26] Jahn L, Hombrink P, Hagedoorn RS, et al. TCR-based therapy for multiple myeloma and other B-cell malignancies targeting intracellular transcription factor BOB1. Blood 2017:129(10):1284-95. 03.

[27] Raje NS, Berdeja JG, Lin Y, et al. bb2121 anti-BCMA CAR T-cell therapy in patients with relapsed/refractory multiple myeloma: Updated results from a multicenter phase I study. N Engl J Med; 2021;384:705-16. https://doi.org/10.1056/NEJMoa2024850.

[28] Ramos CA, Savoldo B, Torrano V, et al. Clinical responses with T lymphocytes targeting malignancy-associated $\kappa$ light chains. J Clin Invest 2016;126(7):2588-96. 07.

[29] Zhao WH, Liu J, Wang BY, et al. A phase 1, open-label study of LCAR-B38M, a chimeric antigen receptor $\mathrm{T}$ cell therapy directed against B cell maturation antigen, in patients with relapsed or refractory multiple myeloma. J Hematol Oncol 2018;11(1):141. 12

[30] Orlando EJ, Han X, Tribouley C, et al. Genetic mechanisms of target antigen loss in CAR19 therapy of acute lymphoblastic leukemia. Nat Med 2018;24(10): 1504-6. 10.

[31] Fischer J, Paret C, El Malki K, et al. CD19 Isoforms Enabling Resistance to CART-19 Immunotherapy Are Expressed in B-ALL Patients at Initial Diagnosis. J Immunother 2017;40(5):187-95. 06.

[32] Sotillo E, Barrett DM, Black KL, et al. Convergence of Acquired Mutations and Alternative Splicing of CD19 Enables Resistance to CART-19 Immunotherapy. Cancer Discov 2015;5(12):1282-95.

[33] Ali SA, Shi V, Maric I, et al. T cells expressing an anti-B-cell maturation antigen chimeric antigen receptor cause remissions of multiple myeloma. Blood 2016;128(13):1688-700. 09

[34] Helsen CW, Hammill JA, Lau VWC, et al. The chimeric TAC receptor co-opts the T cell receptor yielding robust anti-tumor activity without toxicity. Nat Commun 2018;9(1):3049

[35] Rabinovich BA, Ye Y, Etto T, et al. Visualizing fewer than 10 mouse T cells with an enhanced firefly luciferase in immunocompetent mouse models of cancer. Proc Natl Acad Sci U S A 2008;105(38):14342-6.

[36] Helsen CW, Bramson J, Dvorkin-Gheva A, Denisova GF, Bezverbnaya K, Mwawasi KA, T Cell-Antigen Coupler with Y182T Mutation and Methods and Uses Thereof (PCT/CA2018/051290). patent application. https://patentscope.wipo.int/search/ en/detail.jsf?docId=WO2019071358.2019.

[37] Hammill JA, VanSeggelen H, Helsen CW, et al. Designed ankyrin repeat proteins are effective targeting elements for chimeric antigen receptors. J Immunother Cancer 2015;3:55.

[38] Kozakov D, Hall DR, Xia B, et al. The ClusPro web server for protein-protein docking. Nat Protoc 2017;12(2):255-78.

[39] Sobolev V, Sorokine A, Prilusky J, Abola EE, Edelman M. Automated analysis of interatomic contacts in proteins. Bioinformatics 1999;15(4):327-32.

[40] Monaco G, Chen H, Poidinger M, Chen J, de Magalhães JP, Larbi A. flowAI: automatic and interactive anomaly discerning tools for flow cytometry data. Bioinformatics 2016;32(16):2473-80. 08.

[41] Van Gassen S, Callebaut B, Van Helden MJ, et al. FlowSOM: Using self-organizing maps for visualization and interpretation of cytometry data. Cytometry A. Jul 2015;87(7):636-45

[42] Weber LM, Nowicka M, Soneson C, Robinson MD. diffcyt: Differential discovery in high-dimensional cytometry via high-resolution clustering. Commun Biol 2019;2:183.

[43] Amir e-A, Davis KL, Tadmor MD, et al. viSNE enables visualization of high dimensional single-cell data and reveals phenotypic heterogeneity of leukemia. Nat Biotechnol 2013;31(6):545-52.
[44] Crowell H, Zanotelli V, Chevrier S, Robinson M (2021). CATALYST: Cytometry dATa anALYSis Tools. $\mathrm{R}$ package version 1.16.0, https://github.com/HelenaLC/ CATALYST

[45] Bertolet G, Liu D. The Planar Lipid Bilayer System Serves as a Reductionist Approach for Studying NK Cell Immunological Synapses and Their Functions. Methods Mol Biol 2016:1441:151-65.

[46] Schindelin J, Arganda-Carreras I, Frise E, et al. Fiji: an open-source platform for biological-image analysis. Nat Methods 2012;9(7):676-82

[47] Shalaby MR, Shepard HM, Presta L, et al. Development of humanized bispecific antibodies reactive with cytotoxic lymphocytes and tumor cells overexpressing the HER2 protooncogene. J Exp Med 1992;175(1):217-25.

[48] Zhu Z, Carter P. Identification of heavy chain residues in a humanized anti-CD3 antibody important for efficient antigen binding and T cell activation. J Immunol 1995;155(4):1903-10.

[49] Helsen CW, Bramson J, Dvorkin-Gheva A, Denisova GF, Bezverbnaya K, Mwawasi KA, T Cell-Antigen Coupler with Y182T Mutation and Methods and Uses Thereo (WO2019071358). patent application PCT/CA2018/051290. 2019.

[50] Frigault MJ, Lee J, Basil MC, et al. Identification of chimeric antigen receptors that mediate constitutive or inducible proliferation of T cells. Cancer Immunol Res 2015;3(4):356-67.

[51] Gomes-Silva D, Mukherjee M, Srinivasan M, et al. Tonic 4-1BB Costimulation in Chimeric Antigen Receptors Impedes T Cell Survival and Is Vector-Dependent. Cell Rep. 2017;21(1):17-26.

[52] Sakemura R, Terakura S, Watanabe K, et al. A Tet-On Inducible System for Controlling CD19-Chimeric Antigen Receptor Expression upon Drug Administration. Cancer Immunol Res 2016;4(8):658-68. 08.

[53] Ritter AT, Asano Y, Stinchcombe IC, et al Actin depletion initiates events leading to granule secretion at the immunological synapse. Immunity 2015;42(5): $864-76$

[54] Monks CR, Freiberg BA, Kupfer H, Sciaky N, Kupfer A. Three-dimensional segregation of supramolecular activation clusters in T cells. Nature 1998;395(6697): 82-6.

[55] Ashouri JF, Weiss A. Endogenous Nur77 Is a Specific Indicator of Antigen Receptor Signaling in Human T and B Cells. J Immunol 2017;198(2):657-68. 01.

[56] Smith KA. Interleukin-2: inception, impact, and implications. Science 1988;240 (4856):1169-76

[57] Baeuerle PA, Ding J, Patel E, et al. Synthetic TRuC receptors engaging the complete T cell receptor for potent anti-tumor response. Nat Commun 2019;10(1):2087. 05.

[58] Xu Y, Yang Z, Horan LH, et al. A novel antibody-TCR (AbTCR) platform combines Fab-based antigen recognition with gamma/delta-TCR signaling to facilitate Tcell cytotoxicity with low cytokine release. Cell Discov 2018;4:62.

[59] Walter P, Johnson AE. Signal sequence recognition and protein targeting to the endoplasmic reticulum membrane. Annu Rev Cell Biol 1994;10:87-119.

[60] Owji H, Nezafat N, Negahdaripour M, Hajiebrahimi A, Ghasemi Y. A comprehensive review of signal peptides: Structure, roles, and applications. Eur J Cell Biol 2018;97(6):422-41.

[61] Long AH, Haso WM, Shern JF, et al. 4-1BB costimulation ameliorates T cell exhaustion induced by tonic signaling of chimeric antigen receptors. Nat Med 2015;21 (6):581-90.

[62] Eyquem J, Mansilla-Soto J, Giavridis T, et al. Targeting a CAR to the TRAC locus with CRISPR/Cas9 enhances tumour rejection. Nature 2017;543(7643):113-7. 03.

[63] Roybal KT, Rupp LJ, Morsut L, et al. Precision Tumor Recognition by T Cells With Combinatorial Antigen-Sensing Circuits. Cell 2016;164(4):770-9.

[64] Dustin ML. The immunological synapse. Cancer Immunol Res 2014;2(11): 1023-33.

[65] Tsun A, Qureshi I, Stinchcombe JC, et al. Centrosome docking at the immunological synapse is controlled by Lck signaling. J Cell Biol 2011;192(4):663-74.

[66] Labrecque N, Whitfield LS, Obst R, Waltzinger C, Benoist C, Mathis D. How much TCR does a T cell need? Immunity 2001;15(1):71-82.

[67] Perez OD, Mitchell D, Jager GC, et al. Leukocyte functional antigen 1 lowers T cell activation thresholds and signaling through cytohesin- 1 and Jun-activating binding protein 1. Nat Immunol 2003;4(11):1083-92.

[68] Fuller MJ, Zajac AJ. Ablation of CD8 and CD4 T cell responses by high viral loads. J Immunol 2003;170(1):477-86.

[69] Itoh Y, Germain RN. Single cell analysis reveals regulated hierarchical T cell antigen receptor signaling thresholds and intraclonal heterogeneity for individual cytokine responses of CD4+ T cells. J Exp Med 1997;186(5):757-66.

[70] Brown RD, Pope B, Yuen E, Gibson J, DE Joshua. The expression of T cell related costimulatory molecules in multiple myeloma. Leuk Lymphoma 1998;31(34):379-84.

[71] Mozaffari F, Hansson L, Kiaii S, et al. Signalling molecules and cytokine production in T cells of multiple myeloma-increased abnormalities with advancing stage. $\mathrm{Br} \mathrm{J}$ Haematol 2004;124(3):315-24.

[72] Bianchi A, Mariani S, Beggiato E, et al. Distribution of T-cell signalling molecules in human myeloma. Br J Haematol 1997;97(4):815-20. 